\title{
RI/MOM and RI/SMOM renormalization of overlap quark bilinears on domain wall fermion configurations
}

\author{
Yujiang Bi, ${ }^{1, *}$ Hao Cai, ${ }^{1, \dagger}$ Ying Chen, ${ }^{2,3}$ Ming Gong, ${ }^{2,3}$ Keh-Fei Liu, ${ }^{4}$ Zhaofeng Liu, ${ }^{2,3,}$ and Yi-Bo Yang ${ }^{4,5}$ \\ $(\chi Q \mathrm{QCD}$ Collaboration) \\ ${ }^{1}$ School of Physics and Technology, Wuhan University, Wuhan 430072, China \\ ${ }^{2}$ Institute of High Energy Physics and Theoretical Physics Center for Science Facilities, \\ Chinese Academy of Sciences, Beijing 100049, China \\ ${ }^{3}$ School of Physics, University of Chinese Academy of Sciences, Beijing 100049, China \\ ${ }^{4}$ Department of Physics and Astronomy, University of Kentucky, Lexington, Kentucky 40506, USA \\ ${ }^{5}$ Department of Physics and Astronomy, Michigan State University, East Lansing, Michigan 48824, USA
}

(Received 6 November 2017; published 1 May 2018)

\begin{abstract}
Renormalization constants (RCs) of overlap quark bilinear operators on $2+1$-flavor domain wall fermion configurations are calculated by using the RI/MOM and RI/SMOM schemes. The scale independent RC for the axial vector current is computed by using a Ward identity. Then the RCs for the quark field and the vector, tensor, scalar, and pseudoscalar operators are calculated in both the RI/MOM and RI/SMOM schemes. The $\mathrm{RCs}$ are converted to the $\overline{\mathrm{MS}}$ scheme and we compare the numerical results from using the two intermediate schemes. The lattice size is $48^{3} \times 96$ and the inverse spacing $1 / a=1.730(4) \mathrm{GeV}$.
\end{abstract}

DOI: 10.1103/PhysRevD.97.094501

\section{INTRODUCTION}

With the setup of overlap valence on domain wall fermion (DWF) configurations, the $\chi \mathrm{QCD}$ Collaboration has been determining the strangeness and charmness in the nucleon [1], the charm and strange quark masses [2], and other physical quantities of interests. These works are based on RBC-UKQCD DWF configurations with lattice sizes $24^{3} \times 64(24 \mathrm{I})$ and $32^{3} \times 64(32 \mathrm{I})[3,4]$. To shrink uncertainties from chiral extrapolations in calculations at unphysical light quark masses, the RBC-UKQCD Collaborations have generated configurations at the physical pion mass on $48^{3} \times 96$ lattices [5]. On this gauge ensemble labeled as 48I, the $\chi \mathrm{QCD}$ Collaboration is studying the $\rho$ resonance [6], nucleon magnetic moment $[7,8]$, and decay constants of pseudoscalar and vector mesons [9]. To link hadronic matrix elements computed on the lattice to the continuum world, we need the RCs for the corresponding operators. In this paper we present our

\footnotetext{
*biyujiang@ihep.ac.cn

†hcai@whu.edu.cn

tiuzf@ihep.ac.cn
}

Published by the American Physical Society under the terms of the Creative Commons Attribution 4.0 International license. Further distribution of this work must maintain attribution to the author(s) and the published article's title, journal citation, and DOI. Funded by SCOAP ${ }^{3}$. calculation of the RCs for the flavor nonsinglet scalar $(S)$, pseudoscalar $(P)$, vector $(V)$, axial vector $(A)$ and tensor $(T)$ currents of overlap valence quark on the 48I ensemble. The quark field $\mathrm{RC}$ is also obtained.

The RI/MOM scheme [10] is a popular nonperturbative method to calculate RCs in lattice calculations. The results are then converted to the $\overline{\mathrm{MS}}$ scheme by using conversion ratios from perturbation theory. With the shrink of statistical uncertainties in RCs of flavor nonsinglet quark bilinears, the truncation error in the conversion ratio from the RI/MOM scheme to the $\overline{\mathrm{MS}}$ scheme starts to dominate the total uncertainty of the $\mathrm{RC} Z_{S}\left(=1 / Z_{m}\right)$. To reduce this truncation error, the RI/SMOM scheme $[11,12]$ was proposed in which unexceptional or symmetric momentum modes are used when calculating vertex functions of operators. The conversion ratio from the RI/SMOM scheme to the $\overline{\mathrm{MS}}$ scheme for the scalar density was shown to converge much faster than in the case of RI/MOM scheme $[13,14]$. Also, the nonperturbative effects from chiral symmetry breaking and other infrared effects are expected to be more suppressed in the RI/SMOM scheme [11].

In this work we compute the aforementioned RCs by using both the RI/MOM and RI/SMOM schemes. In the end the RCs are converted to the $\overline{\mathrm{MS}}$ scheme. The numerical results are compared to try to see the advantages and shortcomings of the two intermediate schemes. After converting to the $\overline{\mathrm{MS}}$ scheme, we perform perturbative 
runnings and give the results at $2 \mathrm{GeV}$ for the scale dependent RCs $Z_{q}, Z_{S}, Z_{P}$ and $Z_{T}$. Throughout this paper we use the conventions below for the RCs of the quark field, quark mass and bilinear operators:

$$
\psi_{R}=Z_{q}^{1 / 2} \psi_{R}, \quad m_{R}=Z_{m} m_{B}, \quad \mathcal{O}_{R}=Z_{\mathcal{O}} \mathcal{O}_{B},
$$

where the subscripts $R$ and $B$ denote the renormalized and bare quantities respectively.

This paper is organized as follows. In Sec. II we give our framework of the calculation, including the definitions of the renormalization schemes, our overlap fermion Dirac operator and the information of the gauge configurations. Section III shows the computation details, the numerical results and discussions. Finally we summarize in Sec. IV.

\section{FRAMEWORK OF OUR CALCULATION}

In both the RI/MOM and RI/SMOM schemes, the renormalization condition for an operator is imposed on its amputated Green function in the vanishing quark mass limit. The Green function $G_{\mathcal{O}}$ is computed between two external off-shell quark states in Landau gauge. If using a point source quark propagator, one has

$$
G_{\mathcal{O}}\left(p_{1}, p_{2}\right)=\sum_{x, y} e^{-i\left(p_{1} \cdot x-p_{2} \cdot y\right)}\langle\psi(x) \mathcal{O}(0) \bar{\psi}(y)\rangle,
$$

where $\mathcal{O}=\bar{\psi} \Gamma \psi$ with $\Gamma=I, \gamma_{5}, \gamma_{\mu}, \gamma_{\mu} \gamma_{5}, \sigma_{\mu \nu}\left(=\frac{1}{2}\left[\gamma_{\mu}, \gamma_{\nu}\right]\right)$. The amputated Green function is then

$$
\Lambda_{\mathcal{O}}\left(p_{1}, p_{2}\right)=S^{-1}\left(p_{1}\right) G_{\mathcal{O}}\left(p_{1}, p_{2}\right) S^{-1}\left(p_{2}\right),
$$

where the quark propagator $S(p)$ in momentum space is

$$
S(p)=\sum_{x} e^{-i p \cdot x}\langle\psi(x) \bar{\psi}(0)\rangle .
$$

In the RI/MOM scheme, one uses the forward Green function. That is to say, the momenta satisfy $p_{1}=p_{2}=p$. The renormalization condition is imposed at the scale $p_{1}^{2}=p_{2}^{2}=p^{2}=\mu^{2}$ by

$$
\lim _{m_{R} \rightarrow 0} Z_{q}^{-1} Z_{\mathcal{O}} \frac{1}{12} \operatorname{Tr}\left[\Lambda_{\mathcal{O}, B}(p) \Lambda_{\mathcal{O}}^{\text {tree }}(p)^{-1}\right]_{p^{2}=\mu^{2}}=1,
$$

where the subscript $B$ stands for bare and the projector $\Lambda_{\mathcal{O}}^{\text {tree }}(p)=\Gamma$ for the quark bilinears considered in this work. The quark field RC in the RI/MOM scheme is determined by

$$
Z_{q}^{\mathrm{RI} / \mathrm{MOM}}(\mu)=\lim _{m_{R} \rightarrow 0} \frac{-i}{48} \operatorname{Tr}\left[\gamma_{\nu} \frac{\partial S^{-1}(p)}{\partial p_{\nu}}\right]_{p^{2}=\mu^{2}},
$$

which is compatible with the vector Ward-Takahashi identity. To avoid the inconvenience caused by the derivative with respect to the discretized momenta on the lattice, we use the $\mathrm{RC}$ for the local axial vector current $Z_{A}^{\mathrm{RI} / \mathrm{MOM}}$ as the input to calculate other RCs. For example, from Eq. (5) the quark field RC can be obtained by

$$
Z_{q}^{\mathrm{RI} / \mathrm{MOM}}=Z_{A}^{\mathrm{RI} / \mathrm{MOM}} \lim _{m_{R} \rightarrow 0} \frac{1}{12} \operatorname{Tr}\left[\Lambda_{A, B}(p) \Lambda_{A}^{\mathrm{tree}}(p)^{-1}\right]_{p^{2}=\mu^{2}} .
$$

At large $\mu$ the renormalization condition for $Z_{A}^{\mathrm{RI} / \mathrm{MOM}}$ is compatible with the axial vector Ward-Takahashi identity [10]. Thus $Z_{A}^{\mathrm{RI} / \mathrm{MOM}}$ equals to a value $Z_{A}^{\mathrm{WI}}$ obtained from some Ward identity on hadron states. In our work below, we shall use the partially conserved axial current (PCAC) relation to determine $Z_{A}^{\mathrm{WI}}$. We will also use the relation $Z_{A}^{\mathrm{WI}}=Z_{A}^{\overline{\mathrm{MS}}}$ in this work since the $\overline{\mathrm{MS}}$ scheme is consistent with the chiral Ward identities too.

In the RI/SMOM scheme [12], one uses the symmetric momentum configuration

$$
q^{2} \equiv\left(p_{1}-p_{2}\right)^{2}=p_{1}^{2}=p_{2}^{2}=\mu^{2}
$$

when fixing the RCs at the scale $\mu$. The projectors for the amputated Green functions of the scalar, pseudoscalar and tensor currents are the same as those in the RI/MOM scheme. But for the vector and axial vector currents the conditions are [12]

$$
\begin{gathered}
\lim _{m_{R} \rightarrow 0} Z_{q}^{-1} Z_{V} \frac{1}{12 q^{2}} \operatorname{Tr}\left[q_{\mu} \Lambda_{V, B}^{\mu}\left(p_{1}, p_{2}\right) q\right]_{\mathrm{sym}}=1, \\
\lim _{m_{R} \rightarrow 0} Z_{q}^{-1} Z_{A} \frac{1}{12 q^{2}} \operatorname{Tr}\left[q_{\mu} \Lambda_{A, B}^{\mu}\left(p_{1}, p_{2}\right) \gamma_{5} q\right]_{\mathrm{sym}}=1
\end{gathered}
$$

Here the subscript "sym" denotes the symmetric momentum configuration in Eq. (8). The quark field $\mathrm{RC}$ in the $\mathrm{RI} / \mathrm{SMOM}$ scheme is given by

$$
Z_{q}^{\mathrm{RI} / \mathrm{SMOM}}=\lim _{m_{R} \rightarrow 0} \frac{1}{12 p^{2}} \operatorname{Tr}\left[S_{B}^{-1}(p) p\right]_{p^{2}=\mu^{2}},
$$

which is the same as that in the RI'/MOM scheme [10]. The conditions in Eqs. (9), (10), and (11) are compatible with the vector and axial vector Ward-Takahashi identities [12]. Therefore one has $Z_{A}^{\mathrm{RI} / \mathrm{SMOM}}=Z_{A}^{\mathrm{WI}}$. Then by using Eq. (10), we can, alternatively, obtain

$Z_{q}^{\mathrm{RI} / \mathrm{SMOM}}=\lim _{m_{R} \rightarrow 0} Z_{A}^{\mathrm{WI}} \frac{1}{12 q^{2}} \operatorname{Tr}\left[q_{\mu} \Lambda_{A, B}^{\mu}\left(p_{1}, p_{2}\right) \gamma_{5} q\right]_{\mathrm{sym}}$.

In using the RI/MOM and RI/SMOM schemes in practical lattice calculations at a lattice spacing $a$, one needs a renormalization window 


$$
\Lambda_{\mathrm{QCD}} \ll \mu \ll \pi / a,
$$

in which both the infrared effects from chiral symmetry breaking and the ultraviolet effects from the lattice cutoff are small. Also perturbation theory can only apply at large enough momentum scale for calculating the conversion ratios of RCs to the $\overline{\mathrm{MS}}$ scheme.

We use overlap fermions [15] as the valence quark. Our massless overlap operator is given by

$$
D_{o v}(\rho)=1+\gamma_{5} \varepsilon\left(\gamma_{5} D_{\mathrm{w}}(\rho)\right),
$$

where $\varepsilon$ is the matrix sign function and $D_{\mathrm{w}}(\rho)$ is the usual Wilson fermion operator, except with a negative mass parameter $-\rho=1 / 2 \kappa-4$ in which $\kappa_{c}<\kappa<0.25 . \kappa$ is set to 0.2 in our calculation, which corresponds to $\rho=1.5$. The massive overlap Dirac operator is defined as

$$
\begin{aligned}
D_{m} & =\rho D_{o v}(\rho)+m\left(1-\frac{D_{o v}(\rho)}{2}\right) \\
& =\rho+\frac{m}{2}+\left(\rho-\frac{m}{2}\right) \gamma_{5} \varepsilon\left(\gamma_{5} D_{w}(\rho)\right) .
\end{aligned}
$$

To accommodate the SU(3) chiral transformation, we use the chirally regulated field $\hat{\psi}=\left(1-\frac{1}{2} D_{o v}\right) \psi$ in place of $\psi$ in the interpolation field and the currents. This amounts to leave the unmodified currents and instead adopt the effective propagator

$$
G \equiv D_{\text {eff }}^{-1} \equiv\left(1-\frac{D_{o v}}{2}\right) D_{m}^{-1}=\frac{1}{D_{c}+m},
$$

where $D_{c}=\frac{\rho D_{o v}}{1-D_{o v} / 2}$ is chiral, i.e., $\left\{\gamma_{5}, D_{c}\right\}=0$ [16]. With the good chiral properties of overlap fermions, we should expect $Z_{S}=Z_{P}$ and $Z_{V}=Z_{A}$. These relations are indeed satisfied within uncertainties by our numerical results as will be shown later. We also expect that the RI/SMOM results satisfy these relations better than the RI/MOM results since the RI/SMOM scheme suppresses more nonperturbative effects from chiral symmetry breaking.

The gauge configurations that we use in this work are from the RBC-UKQCD Collaborations [5]. 2+1-flavor domain wall fermions were used as the sea quarks in generating these configurations. The light sea quark mass is essentially at the physical point. The lattice size is $48^{3} \times 96$ and the inverse lattice spacing is $1 / a=1.730(4) \mathrm{GeV}$. This ensemble is called 48I by the RBC-UKQCD Collaborations. The parameters of these configurations are collected in Table I.

We may expect that our results of the RCs in the $\mathrm{RI} / \mathrm{MOM}$ and in the $\overline{\mathrm{MS}}$ schemes will be in consistency with our previous calculation of the RCs on the 24I ensemble [17]. The reasons are as follows. The 24I ensemble uses a domain wall fermion with the Shamir
TABLE I. Parameters of configurations with $2+1$-flavor dynamical domain wall fermions (RBC-UKQCD Collaborations [5]). The number of configurations used in this work is 81 . The residual mass is from Fig. 6 in [5].

\begin{tabular}{lccccc}
\hline \hline $1 / a(\mathrm{GeV})$ & Label & $a m_{l}^{\text {sea }} / a m_{s}^{\text {sea }}$ & Volume & $N_{\text {conf }}$ & $a m_{\text {res }}$ \\
\hline $1.730(4)$ & 48I & $0.00078 / 0.0362$ & $48^{3} \times 96$ & 81 & $0.0006102(40)$ \\
\hline \hline
\end{tabular}

kernel and the Iwasaki gauge action. It has a similar lattice spacing as the 48I ensemble, which also uses the Iwasaki gauge action. Although the 48I ensemble uses a domain wall fermion with the Möbius kernel, the Möbius domain wall action is tuned such that the Möbius and Shamir kernels are identical up to a numerical factor [5]. For the valence part, our overlap quark actions are the same on these two ensembles. Although different boundary conditions in the time direction are used on the two ensembles when calculating the quark propagators and this can introduce difference in the RCs as finite-volume effects, we find consistency within uncertainties in the RCs on the two ensembles as we will show later.

In this work we only have one light sea quark mass, and thus cannot extrapolate to the light sea quark massless limit. Our previous final results [17] were given in the chiral limit of the light sea quark mass since the 24I ensemble has three different values for $a m_{l}^{\text {sea }}$, enabling a chiral extrapolation. However we can still compare the results in this work directly with those in [17] since now the light sea quark mass is almost at the physical point, very close to the chiral limit. To estimate the error from not being at $m_{l, s}^{\text {sea }}=0$, we combine our current results at the almost physical $m_{l}^{\text {sea }}$ with our previous results at three larger $m_{l}^{\text {sea }}$ 's to estimate the light sea quark mass dependence. Then from this dependence we estimate the error due to $m_{s}^{\text {sea }} \neq 0$.

In Table II we give the overlap valence quark masses in lattice units used in this work. The corresponding pion masses in the table were measured in Ref. [6] and are close to the chiral limit. The precise values of the pion masses do not matter here since we will use the valence quark mass to do the extrapolations to the chiral limit. At the three lightest valence quark masses finite-volume effects may become big since $m_{\pi} L<4$. We will check these effects at below as we study the systematic uncertainties of our RCs.

\section{CALCULATION AND NUMERICAL RESULTS}

We use periodic boundary conditions in all four directions. Therefore the discretized momenta in lattice units are

$$
a p=2 \pi\left(\frac{k_{1}}{L}, \frac{k_{2}}{L}, \frac{k_{3}}{L}, \frac{k_{4}}{T}\right),
$$


TABLE II. Overlap valence quark masses in lattice units used in this work. The corresponding pion masses are from Ref. [6].

\begin{tabular}{lcccccccc}
\hline \hline$a m_{q}$ & 0.00120 & 0.00170 & 0.00240 & 0.00300 & 0.00455 & 0.00600 & 0.0102 & 0.0203 \\
\hline$m_{\pi} / \mathrm{MeV}$ & $95(3)$ & $114(2)$ & $135(2)$ & $149(2)$ & $182(2)$ & $208(2)$ & $267(1)$ & $371(1)$ \\
\hline \hline
\end{tabular}

where $L=48, T=96$ and $k_{\mu}$ are integers. In doing the Fourier transformation for the point source quark propagators, we set $k_{\mu}=-12,-11, \ldots, 12$. To reduce the effects of Lorentz noninvariant discretization errors, we only use the momenta which satisfy the "democratic" condition

$$
\frac{p^{[4]}}{\left(p^{2}\right)^{2}}<0.29, \text { where } p^{[4]}=\sum_{\mu} p_{\mu}^{4}, \quad p^{2}=\sum_{\mu} p_{\mu}^{2}
$$

in performing the RI/MOM scheme calculation. In other words, only those momenta aligning along or close to the 4-dimensional diagonal line are used for the RI/MOM analyses. For the RI/SMOM calculation, the conditions in Eq. (8) cannot be easily satisfied. Thus we do not apply any "democratic" cuts like the one in Eq. (18) for this case. We use point source quark propagators in Landau gauge to compute all the necessary gauge dependent Green functions and vertex functions. The statistical errors of our numerical results are from Jackknife processes with one configuration removed each time.

After obtaining the RCs in the RI/MOM and RI/SMOM schemes at each scale $\mu$, we convert them to their $\overline{\mathrm{MS}}$ values by using the corresponding conversion ratios calculated in perturbation theory at that scale. Then a perturbative running to $2 \mathrm{GeV}$ in the $\overline{\mathrm{MS}}$ scheme is performed for each RC by using the appropriate anomalous dimensions.

\section{A. Renormalization of the axial vector current from PCAC}

Similar to what was done in Ref. [17], we use the PCAC relation

$$
Z_{A} \partial_{\mu} A_{\mu}=2 Z_{m} m_{q} Z_{P} P
$$

and $Z_{m}=Z_{P}^{-1}$ for overlap fermions to obtain $Z_{A}^{\mathrm{WI}}$. By sandwiching both sides of Eq. (19) into the vacuum and a pion state at rest, one finds

$$
Z_{A}^{\mathrm{WI}}=\frac{2 m_{q}\langle\Omega|P| \pi\rangle}{m_{\pi}\left\langle\Omega\left|A_{4}\right| \pi\right\rangle} .
$$

To get the ratio of matrix elements and the pion mass, we calculate zero momentum 2-point correlators $\quad C_{P P}(t) \equiv \sum_{\vec{x}}\left\langle\Omega\left|P(x) P^{\dagger}(0)\right| \Omega\right\rangle \quad$ and $\quad C_{A_{4} P}(t) \equiv$ $\sum_{\vec{x}}\left\langle\Omega\left|A_{4}(x) P^{\dagger}(0)\right| \Omega\right\rangle$ in practice by using wall-source quark propagators. For a given $m_{q}$, we simultaneously fit the two wall-source point-sink correlators at large source-sink time separation by a single exponential with $m_{\pi}$ as a common parameter. The ratio of the matrix elements is then given by the ratio of the spectral weights (the other two fitting parameters) in front of the exponentials. We make sure the simultaneous fittings have $\chi^{2} /$ d.o.f. $<1.1$.

The resulted $Z_{A}^{\mathrm{WI}}$ is plotted as a function of $a m_{q}$ in Fig. 1, in which we also plot the linear extrapolation of $Z_{A}^{\mathrm{WI}}$ to the chiral limit. The numerical values of $Z_{A}^{\mathrm{WI}}$ are given in Table III. By using all the 9 data points at nonzero $a m_{q}$ in Table III we obtain $Z_{A}^{\mathrm{WI}}=1.1025(8)$ in the chiral limit, where the error is only statistical. One systematic error of $Z_{A}^{\mathrm{WI}}$ in the chiral limit is determined by varying the range of $a m_{q}$ in doing the chiral extrapolation. Using the data points at $a m_{q}>0.004$ (finite volume effects may be large at the four smallest quark masses), we get $Z_{A}^{\mathrm{WI}}=1.1027(11)$. If we drop the data at the largest two quark masses $a m_{q}=$ 0.00160 and 0.02030 , then we find $Z_{A}^{\mathrm{WI}}=1.1023(13)$. In the end we assign the largest difference 0.0004 in these center values as one systematic error. At $1.4 \sigma$ the result $Z_{A}^{\mathrm{WI}}=1.1025(8)(4)$ agrees with $Z_{A}^{\mathrm{WI}}=1.111(6)$ on the 24I ensemble from our previous work [17]. If we combine $Z_{A}^{\mathrm{WI}}=1.1025(8)$ with our three $Z_{A}^{\mathrm{WI}}$ 's at difference light sea quark masses on the 24I ensemble and do a linear chiral extrapolation in $\left(a m_{l}^{\text {sea }}+a m_{\text {res }}\right)$, then we get $Z_{A}^{\mathrm{WI}}=1.1025(9)$ at $a m_{l}^{\text {sea }}+a m_{\text {res }}=0$. Thus we think the error from the light sea quark mass dependence is negligible. The slope from this linear extrapolation is 0.07(17). We use half of this slope (since there is only one flavor of strange quark) to estimate the effect from the nonzero

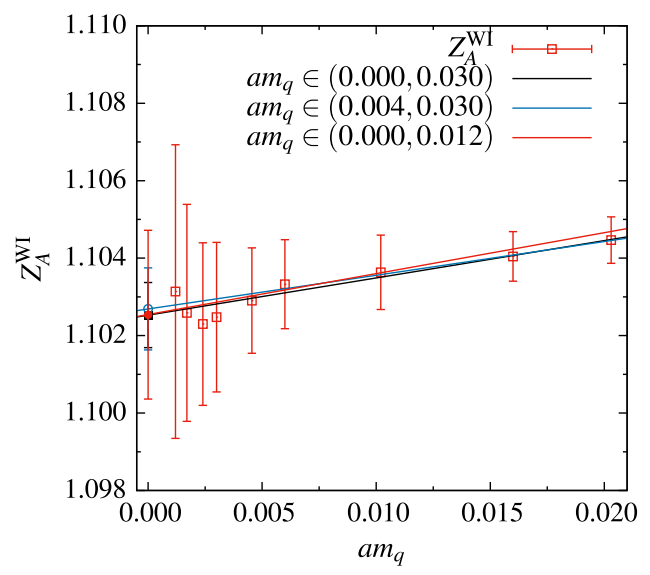

FIG. 1. $Z_{A}^{\mathrm{WI}}$ as a function of the valence quark mass and its linear chiral extrapolations using three different fitting ranges. 
TABLE III. $\quad Z_{A}^{\mathrm{WI}}$ for 9 valence quark masses. $Z_{A}^{\mathrm{WI}}$ at $a m_{q}=0$ is from a linear extrapolation in $a m_{q}$. The first error is statistical, the second is a systematic error from varying the range of $a m_{q}$ in doing the linear chiral extrapolation, the third is from the nonzero strange sea quark mass.

\begin{tabular}{llllll}
\hline \hline$a m_{q}$ & 0.0 & 0.00120 & 0.00170 & 0.00240 & 0.00300 \\
$Z_{A}^{\mathrm{WI}}$ & $1.1025(8)(4)(13)$ & $1.1031(38)$ & $1.1026(28)$ & $1.1023(21)$ & $1.1025(19)$ \\
$a m_{q}$ & 0.00455 & 0.00600 & 0.01020 & 0.01600 & 0.02030 \\
$Z_{A}^{\mathrm{WI}}$ & $1.1029(14)$ & $1.1033(12)$ & $1.1036(10)$ & $1.1041(6)$ & $1.1045(6)$ \\
\hline \hline
\end{tabular}

strange sea quark mass. Multiplying it with $\left(a m_{s}^{\text {sea }}+a m_{\text {res }}\right)=$ 0.0368102 , we obtain an error of 0.0013 as the third error given in Table III. In the analyses below, we use this method to estimate the error from the strange sea quark mass dependence for all RCs except $Z_{T}$, for which we do not have results on the $24 \mathrm{I}$ ensemble.

The contamination from excited states should be negligible in the fittings of the correlators since the pions are pseudo-Goldstone bosons from chiral symmetry breaking and thus have a tiny mass, which is much lighter than those of the other pseudoscalar mesons.

The PCAC relation equation (19) can be imposed at the level of correlation functions as well as the hadronic matrix elements. This gives us another way to calculate $Z_{A}$ and check its systematic errors from finite-volume and excitedstate effects. From Eq. (19) we have

$\sum_{\vec{x}}\left\langle\Omega\left|Z_{A} \partial_{\mu} A_{\mu}(x) P^{\dagger}(0)\right| \Omega\right\rangle=2 m_{q} \sum_{\vec{x}}\left\langle\Omega\left|P(x) P^{\dagger}(0)\right| \Omega\right\rangle$.

Thus

$$
Z_{A}=\frac{4 m_{q} C_{P P}(t)}{C_{A_{4} P}(t+1)-C_{A_{4} P}(t-1)},
$$

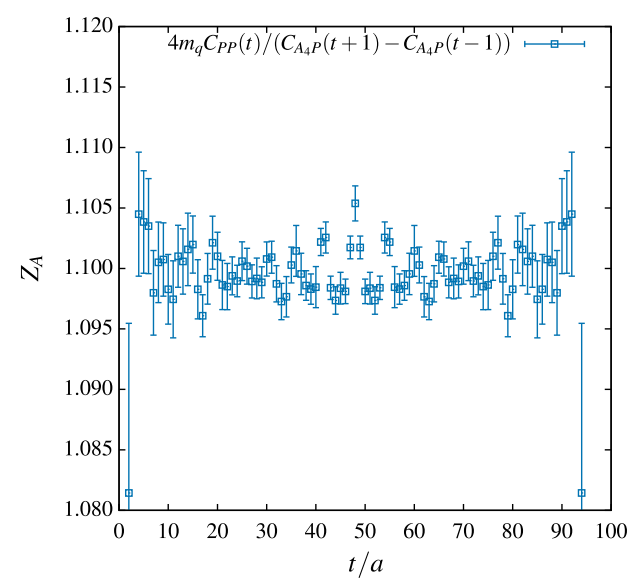

where we have replaced the partial derivative by a difference. The results of $Z_{A}$ obtained in this way are shown in Fig. 2.

In the left panel of Fig. 2, we show $Z_{A}$ from Eq. (22) at the valence quark mass $a m_{q}=0.01020$ as an example. The results at other quark masses are similar. Since we have averaged the correlator $C_{P P}(t)$ (also $C_{A_{4} P}(t)$ ) along the forward and backward time directions, the plateau from the ratio is symmetric about $t=T / 2$. A constant fit in the range $t \in[16,32]$ gives $Z_{A}=1.0995(12)$.

In the right panel of Fig. 2, we show $Z_{A}$ as a function of the valence quark mass. The squares are repeats of Fig. 1, that is to say, the results from Eq. (20). The diamonds are from the ratio of correlators Eq. (22). The two straight lines are linear extrapolations using all data points from the two methods, respectively. In the chiral limit of the valence quark, the two methods give consistent results as shown by the two crosses at $a m_{q}=0$. At the four smallest quark masses, the squares and the diamonds are in good agreement. This confirms that the possible finite-volume effect in $Z_{A}$ from Eq. (20) at small quark masses is small (we estimated the associated error to be 0.0004 in Table III). At large quark masses $a m_{q}>0.006$, the results from Eq. (20) and Eq. (22) are different. This difference we think is from the discretization effects in Eq. (22) as the derivative is replaced by a finite difference on the lattice. To see it, we

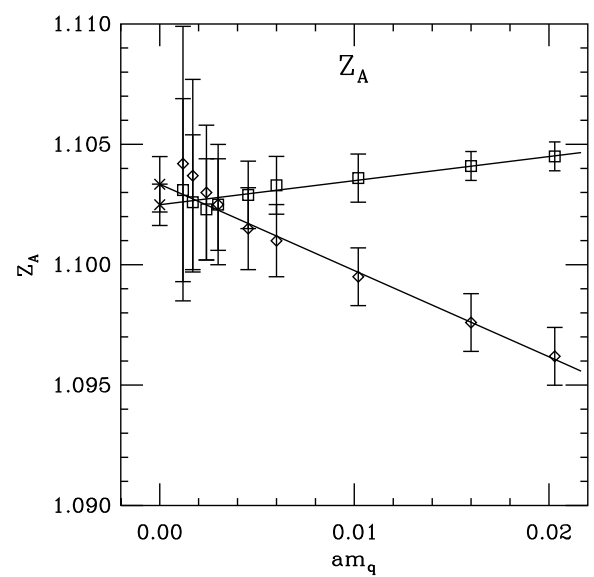

FIG. 2. Left panel: $Z_{A}$ from Eq. (22) at $a m_{q}=0.01020$ as an example. Right panel: Linear chiral extrapolations of $Z_{A}$ from using the hadronic matrix elements (squares) or correlation functions (diamonds). 

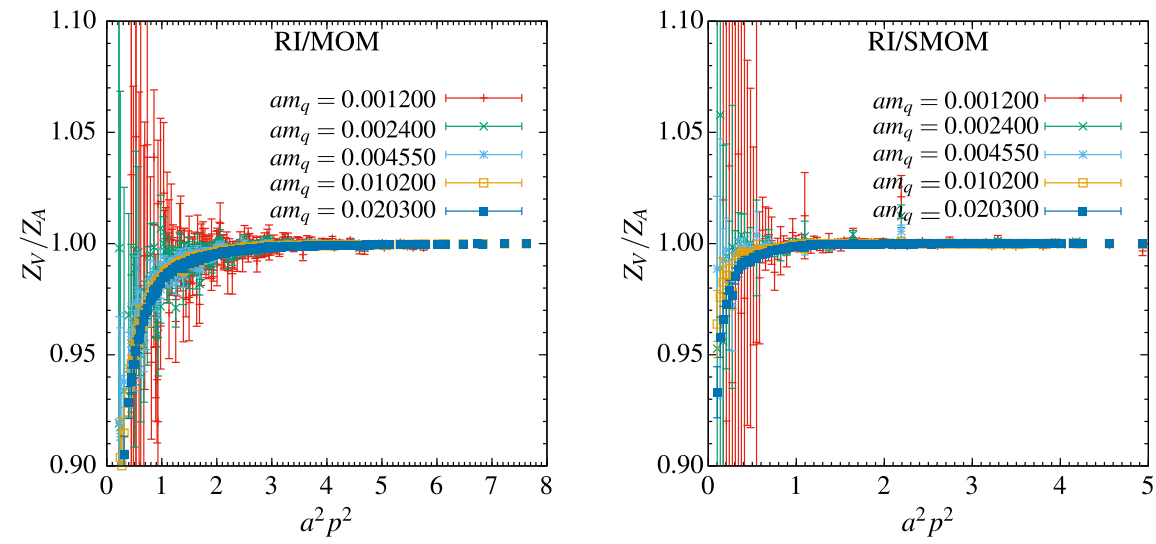

FIG. 3. $Z_{V} / Z_{A}$ in the RI/MOM (left graph) and RI/SMOM (right graph) scheme. The results show little valence quark mass dependence.

consider the large time behavior of $C_{P P}(t)$ and $C_{A_{4} P}(t)$. At large $t, C_{P P}(t)=A \exp \left(-m_{\pi} t\right)+A \exp \left(-m_{\pi}(T-t)\right)$ and $C_{A_{4} P}(t)=-B \exp \left(-m_{\pi} t\right)+B \exp \left(-m_{\pi}(T-t)\right)$. Here $A \propto\langle\Omega|P| \pi\rangle$ and $B \propto\left\langle\Omega\left|A_{4}\right| \pi\right\rangle$. Substituting them into Eq. (22), we get

$Z_{A}=\frac{-4 A m_{q}}{B\left[e^{-m_{\pi}}-e^{m_{\pi}}\right]}=\frac{2 A m_{q}}{B m_{\pi}}\left(1-a^{2} m_{\pi}^{2} / 6+\mathcal{O}\left(a^{4} m_{\pi}^{4}\right)\right)$.

From Table II our $a m_{\pi}$ is around 0.154 at $a m_{q}=0.01020$. Thus $1-a^{2} m_{\pi}^{2} / 6=0.996$ and it explains the difference between $Z_{A}=1.1036(10)$ and 1.0995(12) from the two methods at this quark mass. The difference in $Z_{A}$ at other large quark masses can also be explained by this factor $\left(1-a^{2} m_{\pi}^{2} / 6\right)$.

Since the extrapolation of the squares in the right panel of Fig. 2 has a smaller slope and the two methods give consistent results, we take the numbers in Table III as our final results for $Z_{A}^{\mathrm{WI}}$. Combining all the errors quadratically, we get $Z_{A}^{\mathrm{WI}}=1.1025(16)$.

\section{B. The vector current}

For overlap fermions, $Z_{V}=Z_{A}$ is expected from its good chiral property. We calculate the ratio $Z_{V} / Z_{A}$ in both RI/MOM and RI/SMOM schemes:

$$
\begin{gathered}
\frac{Z_{V}^{\mathrm{RI} / \mathrm{MOM}}}{Z_{A}^{\mathrm{RI} / \mathrm{MOM}}}=\left.\frac{\Gamma_{A}(p)}{\Gamma_{V}(p)}\right|_{p^{2}=\mu^{2}}, \\
\frac{Z_{V}^{\mathrm{RI} / \mathrm{SMOM}}}{Z_{A}^{\mathrm{RI} / \mathrm{SMOM}}}=\left.\frac{\Gamma_{A}\left(p_{1}, p_{2}\right)}{\Gamma_{V}\left(p_{1}, p_{2}\right)}\right|_{\text {sym }},
\end{gathered}
$$

where

$$
\begin{aligned}
& \Gamma_{V}(p)=\frac{1}{48} \operatorname{Tr}\left[\Lambda_{V, B}^{\mu}(p) \gamma_{\mu}\right], \\
& \Gamma_{A}(p)=\frac{1}{48} \operatorname{Tr}\left[\Lambda_{A, B}^{\mu}(p) \gamma_{5} \gamma_{\mu}\right],
\end{aligned}
$$

$$
\begin{aligned}
& \Gamma_{V}\left(p_{1}, p_{2}\right)=\frac{1}{12 q^{2}} \operatorname{Tr}\left[q_{\mu} \Lambda_{V, B}^{\mu}\left(p_{1}, p_{2}\right) q\right] \\
& \Gamma_{A}\left(p_{1}, p_{2}\right)=\frac{1}{12 q^{2}} \operatorname{Tr}\left[q_{\mu} \Lambda_{A, B}^{\mu}\left(p_{1}, p_{2}\right) \gamma_{5} q\right] .
\end{aligned}
$$

The numerical results of this ratio are shown in Fig. 3 for some of the valence quark masses $a m_{q}$. Clearly little quark mass dependence is seen in these results. We do linear extrapolations in $a m_{q}$ for $Z_{V} / Z_{A}$ in both schemes to reach the chiral limit. The comparison of this ratio in the two schemes in the chiral limit is shown in Fig. 4. $Z_{V} / Z_{A}=1$ is well satisfied in both schemes at large momentum scale.

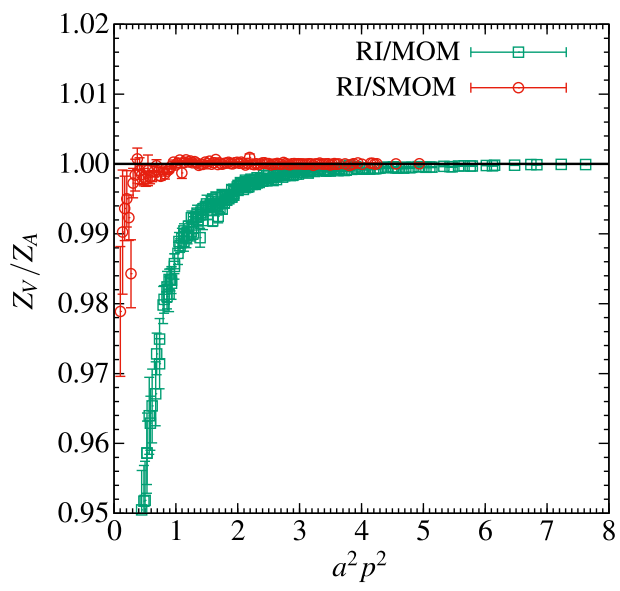

FIG. 4. $Z_{V} / Z_{A}$ in the chiral limit in the two schemes. $Z_{V} / Z_{A}=1$ is well satisfied in both schemes at large momentum scale. This relation is verified at lower momentum scale in the RI/SMOM scheme than in the RI/MOM scheme. The horizontal line is $Z_{V} / Z_{A}=1$ for guiding the eyes. 

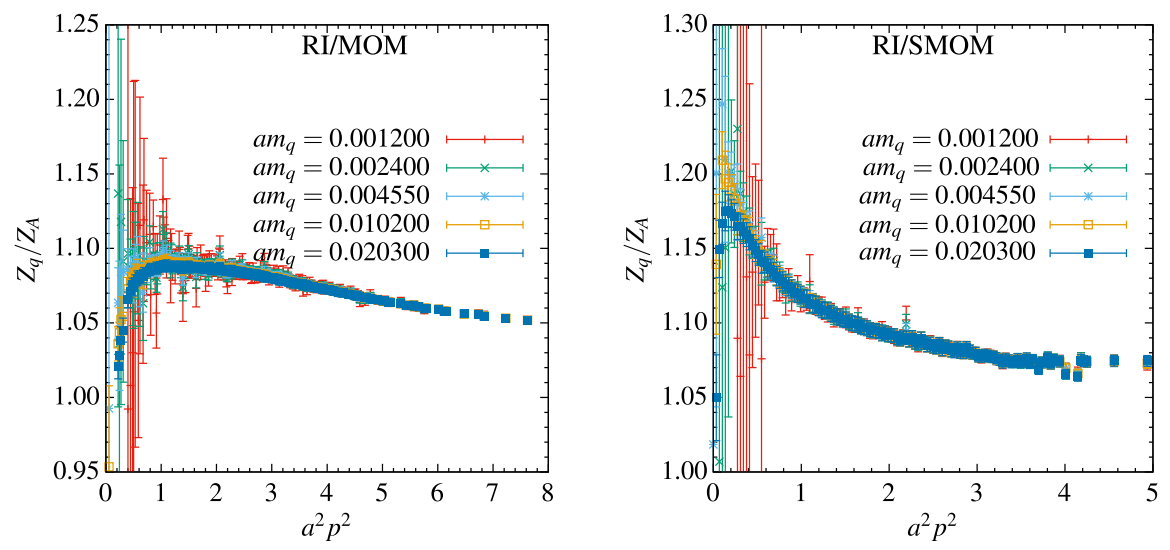

FIG. 5. $Z_{q} / Z_{A}$ in the RI/MOM and RI/SMOM schemes at various valence quark masses.

The RI/SMOM scheme is supposed to have less infrared effects. Indeed, $Z_{V} / Z_{A}=1$ is verified at lower momentum scale in the RI/SMOM scheme than in the RI/MOM scheme as shown in Fig. 4.

\section{Quark field renormalization}

The quark field RC can be used, for example, in analyzing the scalar dressing function of the quark propagator in Landau gauge to determine the quark chiral condensate [18]. After finding $Z_{A}^{\mathrm{WI}}$, we use Eqs. (7) and (12) to calculate $Z_{q}$ in the RI/MOM and RI/SMOM scheme respectively. What we get are shown in Fig. 5, in which $Z_{q} / Z_{A}$ is plotted as a function of the renormalization scale for various valence quark masses. Apparently the quark mass dependence of $Z_{q} / Z_{A}$ in both schemes is very small. The chiral extrapolation can be done with a linear function

$$
\frac{Z_{q}}{Z_{A}}\left(a m_{q}\right)=\frac{Z_{q}}{Z_{A}}+A \cdot a m_{q} .
$$

Examples of this linear extrapolation of $Z_{q} / Z_{A}$ in the $\mathrm{RI} / \mathrm{MOM}$ and RI/SMOM schemes are shown in the two graphs in Fig. 6.

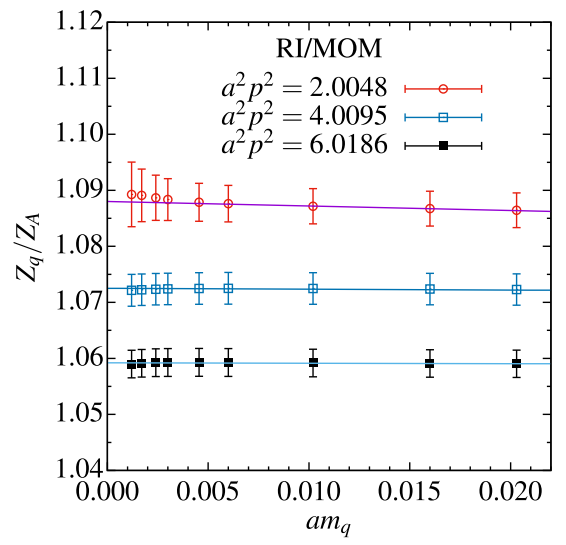

The chiral limit value of $Z_{q}^{\mathrm{RI} / \mathrm{MOM}} / Z_{A}$ can be converted to the $\overline{\mathrm{MS}}$ value $Z_{q}^{\overline{\mathrm{MS}}} / Z_{A}$ by using the following three loop conversion ratio given in Ref. [19] (we use the relation $Z_{A}^{\mathrm{WI}}=Z_{A}^{\mathrm{RI} / \mathrm{MOM}}=Z_{A}^{\mathrm{RI} / \mathrm{SMOM}}=Z_{A}^{\overline{\mathrm{MS}}}$ here and in the rest of the paper)

$$
\begin{aligned}
\frac{Z_{q}^{\overline{\mathrm{MS}}}}{Z_{q}^{\mathrm{R} / \mathrm{MOM}}}= & 1+\left[-\frac{517}{18}+12 \zeta_{3}+\frac{5}{3} n_{f}\right]\left(\frac{\alpha_{s}}{4 \pi}\right)^{2} \\
& +\left[-\frac{1287283}{648}+\frac{14197}{12} \zeta_{3}+\frac{79}{4} \zeta_{4}\right. \\
& \left.-\frac{1165}{3} \zeta_{5}+\frac{18014}{81} n_{f}-\frac{368}{9} \zeta_{3} n_{f}-\frac{1102}{243} n_{f}^{2}\right] \\
& \times\left(\frac{\alpha_{s}}{4 \pi}\right)^{3}+\mathcal{O}\left(\alpha_{s}^{4}\right) .
\end{aligned}
$$

Here $n_{f}$ is the number of flavors and $\zeta_{n}$ is the Riemann zeta function evaluated at $n$. The strong coupling constant $\alpha_{s}(\mu)$ is evaluated in the $\overline{\mathrm{MS}}$ scheme by using its perturbative running to four loops [20]. The beta functions in the $\overline{\mathrm{MS}}$ scheme to four loops can be found in Ref. [21].

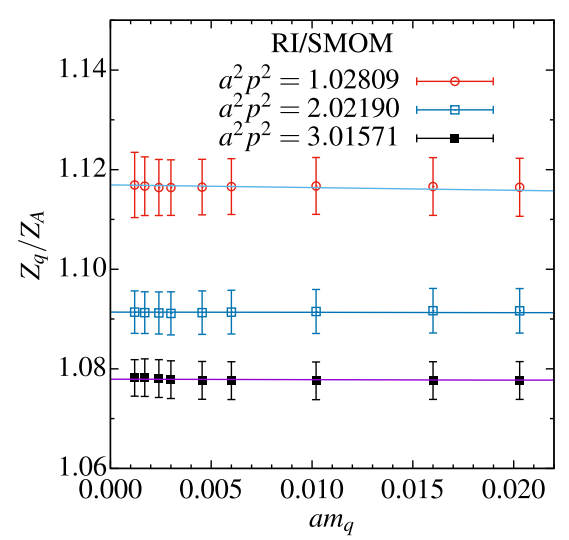

FIG. 6. Linear chiral extrapolations of $Z_{q} / Z_{A}$ in the RI/MOM and RI/SMOM schemes at three typical momentum values. 

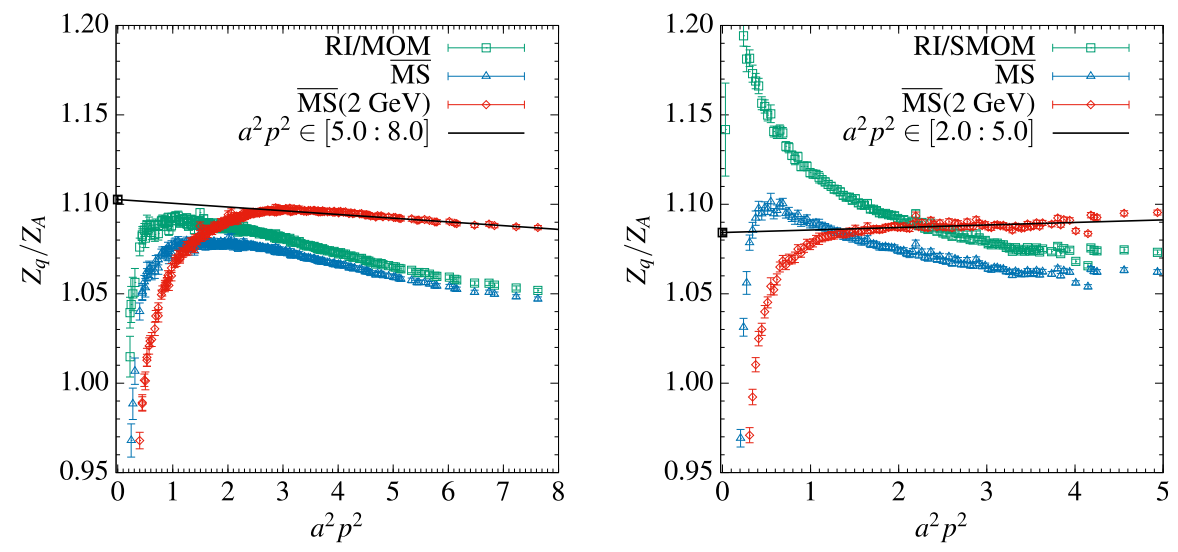

FIG. 7. Conversion of $Z_{q} / Z_{A}$ in the RI/MOM or RI/SMOM scheme to the $\overline{\mathrm{MS}}$ scheme. The running to $2 \mathrm{GeV}$ in the $\overline{\mathrm{MS}}$ scheme is also shown by the red diamonds in both graphs. The black line in each graph is a linear extrapolation in $a^{2} p^{2}$ using data in the indicated range.

And we use $\Lambda_{\mathrm{QCD}}^{\overline{\mathrm{MS}}}=332(17) \mathrm{MeV}$ for three flavors in the $\overline{\mathrm{MS}}$ scheme [22].

Since $Z_{q}^{\mathrm{RI} / \mathrm{SMOM}}=Z_{q}^{\mathrm{RI}^{\prime} / \mathrm{MOM}}$, the conversion of $Z_{q}^{\mathrm{RI} / \mathrm{SMOM}} / Z_{A}$ to its $\overline{\mathrm{MS}}$ value is done with the ratio [19]

$$
\begin{aligned}
\frac{Z_{q}^{\overline{\mathrm{MS}}}}{Z_{q}^{\mathrm{RI} / \mathrm{MOM}}}= & 1+\left[-\frac{359}{9}+12 \zeta_{3}+\frac{7}{3} n_{f}\right]\left(\frac{\alpha_{s}}{4 \pi}\right)^{2} \\
& +\left[-\frac{439543}{162}+\frac{8009}{6} \zeta_{3}+\frac{79}{4} \zeta_{4}\right. \\
& \left.-\frac{1165}{3} \zeta_{5}+\frac{24722}{81} n_{f}-\frac{440}{9} \zeta_{3} n_{f}-\frac{1570}{243} n_{f}^{2}\right] \\
& \times\left(\frac{\alpha_{s}}{4 \pi}\right)^{3}+\mathcal{O}\left(\alpha_{s}^{4}\right) .
\end{aligned}
$$

The conversions of $Z_{q}^{\mathrm{RI} / \mathrm{MOM}} / Z_{A}$ and $Z_{q}^{\mathrm{RI} / \mathrm{SMOM}} / Z_{A}$ are plotted in the two graphs respectively in Fig. 7. The Green squares are the results in the momentum subtraction schemes. After converting them to the $\overline{\mathrm{MS}}$ scheme, we obtain the blue triangles.

Note the perturbative truncation error in the conversion ratio equation (29) for the RI/SMOM scheme is large than that in Eq. (28) for the RI/MOM scheme. For example, at $p^{2}=\mu^{2}=16 \mathrm{GeV}^{2}$ (or $a^{2} p^{2}=5.346$ with our lattice spacing) the numerical value of Eq. (28) can be broken into

$$
\begin{aligned}
& \frac{Z_{q}^{\overline{\mathrm{MS}}}}{Z_{q}^{\mathrm{RI} / \mathrm{MOM}}}\left(\mu=4 \mathrm{GeV}, n_{f}=3\right) \\
& \quad=1-0.0 \alpha_{s}-0.0589 \alpha_{s}^{2}-0.2352 \alpha_{s}^{3}+\cdots \\
& \quad=1-0.0-0.0028-0.0025+\cdots,
\end{aligned}
$$

where we have used $\alpha_{s}^{\overline{\mathrm{MS}}}(4 \mathrm{GeV})=0.2189$. Assuming the coefficient of the $\mathcal{O}\left(\alpha_{s}^{4}\right)$ term is $4(\approx 0.2352 / 0.0589)$ times larger than that of the $\mathcal{O}\left(\alpha_{s}^{3}\right)$ term, we can estimate the $\mathcal{O}\left(\alpha_{s}^{4}\right)$ term to be of size $\sim 0.0022$. This means the truncation error is of size $0.2 \%$. At the same scale, the numerical value of Eq. (29) is

$$
\begin{aligned}
& \frac{Z_{q}^{\overline{\mathrm{MS}}}}{Z_{q}^{\mathrm{RI} / \mathrm{MOM}}}\left(\mu=4 \mathrm{GeV}, n_{f}=3\right) \\
& \quad=1-0.0 \alpha_{s}-0.1169 \alpha_{s}^{2}-0.4076 \alpha_{s}^{3}+\cdots \\
& \quad=1-0.0-0.0056-0.0043+\cdots
\end{aligned}
$$

Assuming the coefficient of the $\mathcal{O}\left(\alpha_{s}^{4}\right)$ term is $3.5(\approx 0.4076 / 0.1169)$ times larger than that of the $\mathcal{O}\left(\alpha_{s}^{3}\right)$ term, we find that the size of the $\mathcal{O}\left(\alpha_{s}^{4}\right)$ term is about 0.0033 . Thus the truncation error is $0.3 \%$.

Our RI/SMOM scheme data do not reach beyond the scale $a^{2} p^{2}=\sim 5$ as shown in the right panel of Fig. 7. We use the data starting from $a^{2} p^{2}=2$ (or $p=2.447 \mathrm{GeV}$ ) in our analyses below. The truncation error in Eq. (29) at $a^{2} p^{2}=2$ can be estimated similarly and its size is $0.7 \%$.

The $\overline{\mathrm{MS}}$ value $Z_{q}^{\overline{\mathrm{MS}}} / Z_{A}$ at a given scale $a^{2} p^{2}$ can be run to $2 \mathrm{GeV}$ by using the quark field anomalous dimension $\gamma_{q}^{\overline{\mathrm{MS}}}$. In perturbation theory $\gamma_{q}^{\overline{\mathrm{MS}}}$ has been calculated to four loops in Landau gauge [23]. The red diamonds in both graphs of Fig. 7 show $Z_{q}^{\overline{\mathrm{MS}}} / Z_{A}\left(2 \mathrm{GeV} ; a^{2} p^{2}\right)$ after the running as a function of the initial scale $a^{2} p^{2}$. From the linear dependence on $a^{2} p^{2}$ at large scale we extrapolate $Z_{q}^{\overline{\mathrm{MS}}} / Z_{A}\left(2 \mathrm{GeV} ; a^{2} p^{2}\right)$ to $a^{2} p^{2}=0$ to remove the $\mathcal{O}\left(a^{2} p^{2}\right)$ lattice artifacts.

In the left graph of Fig. 7 using the RI/MOM scheme, we do the linear extrapolation in the range $a^{2} p^{2} \in[5,8]$ and find $Z_{q}^{\overline{\mathrm{MS}}} / Z_{A}(2 \mathrm{GeV})=1.1027(20)$. If using the range $a^{2} p^{2} \in[4,8]$, then we get $Z_{q}^{\overline{\mathrm{MS}}} / Z_{A}(2 \mathrm{GeV})=1.1052(11)$. The change in the center value $(0.2 \%)$ is taken as a 
systematic error. From our previous work at three light sea quark masses on the 24I ensemble [17,18], we can obtain $Z_{q}^{\overline{\mathrm{MS}}} / Z_{A}(2 \mathrm{GeV})=1.079(18)$ in the light sea quark massless limit, which agrees with our current result 1.1027(20) on the $48 \mathrm{I}$ ensemble within $1.3 \sigma$.

For the right graph of Fig. 7 using the RI/SMOM scheme, we use the range $a^{2} p^{2} \in[2,5]$ for the extrapolation and find $Z_{q}^{\overline{\mathrm{MS}}} / Z_{A}(2 \mathrm{GeV})=1.0842(13)$. The $\chi^{2} /$ d.o.f. of this extrapolation is 2.2 . In the RI/SMOM scheme there is no "democratic" cut on the momenta $p_{1}, p_{2}$ and $q$. Lattice artifacts proportional to $a^{2} p^{[4]} / p^{2}$ make the data points scatter around the smooth curve in $a^{2} p^{2}$ and render the $\chi^{2} /$ d.o.f. big. Thus we enlarge the statistical error from the linear fitting by the factor $\sqrt{\chi^{2} / \text { d.o.f. }}$ to include this uncertainty. In the following analyses for all RCs we similarly inflate the statistical error if the $\chi^{2} /$ d.o.f. of the $a^{2} p^{2}$ extrapolation is larger than 1 . If using the range $a^{2} p^{2} \in[1.5,5]$ or $[2.5,5]$, then we obtain $Z_{q}^{\overline{\mathrm{MS}}} / Z_{A}(2 \mathrm{GeV})=1.0839(9)$ or $1.0818(20)$ respectively. The center value changes by 0.0024 , which is around $0.2 \%$.

Besides the truncation uncertainty in the conversion ratio and the uncertainty from the fitting range of $a^{2} p^{2}$ in the linear extrapolation, we also consider the uncertainties from the lattice spacing, which is needed to determine the value of $a^{2} p^{2}$ corresponding to $p=2 \mathrm{GeV}$, from $\Lambda_{\mathrm{QCD}}^{\overline{\mathrm{MS}}}$, from the perturbative running in the $\overline{\mathrm{MS}}$ scheme, and from the nonzero strange sea quark mass. For the calculation using $\mathrm{RI} / \mathrm{MOM}$ as the intermediate scheme, varying $1 / a=$ $1.730(4) \mathrm{GeV}$ in one sigma leads to $0.2 \%$ change in $Z_{q}^{\overline{\mathrm{MS}}} / Z_{A}(2 \mathrm{GeV})$. Changing $\Lambda_{\mathrm{QCD}}^{\overline{\mathrm{MS}}}=332(17) \mathrm{GeV}$ in one sigma leads to $0.1 \%$ change in $Z_{q}^{\overline{\mathrm{MS}}} / Z_{A}(2 \mathrm{GeV})$. The perturbative running to $2 \mathrm{GeV}$ of $Z_{q}^{\overline{\mathrm{MS}}} / Z_{A}\left(a^{2} p^{2}\right)$ uses four-loop results of the anomalous dimension. The $\mathcal{O}\left(\alpha_{s}^{4}\right)$ term is found to be around $0.2 \%$ of the total size of the running from $a^{2} p^{2}>5$ to $2 \mathrm{GeV}$. Combining $Z_{q}^{\overline{\mathrm{MS}}} / Z_{A}(2 \mathrm{GeV})=1.1027(20)$ on the $48 \mathrm{I}$ ensemble with our previous results on the $24 \mathrm{I}$ ensemble at three light sea quark masses $[17,18]$ for a linear chiral extrapolation, we get $Z_{q}^{\overline{\mathrm{MS}}} / Z_{A}(2 \mathrm{GeV})=1.1030(21)$ in the light sea quark massless limit. It agrees well with 1.1027(20), indicating a small light sea quark mass effect in our current result. The slope from this extrapolation is -1.13(26), from which we find an error of 0.021 due to the strange sea quark mass dependence. The uncertainties of $Z_{q}^{\overline{\mathrm{MS}}} / Z_{A}(2 \mathrm{GeV})$ are listed in Table IV.

Similarly we do the analyses for the calculation using $\mathrm{RI} / \mathrm{SMOM}$ as the intermediate scheme. Since we did not do RI/SMOM calculation on the 24I ensemble, we use the valence quark mass dependence as shown in Fig. 6 to estimate the error from the nonzero strange sea quark mass. The chiral extrapolation in the right panel of Fig. 6 is quite
TABLE IV. Uncertainties of $Z_{q}^{\overline{\mathrm{MS}}} / Z_{A}(2 \mathrm{GeV})$ in the chiral limit. The second and third columns are for using the RI/MOM and $\mathrm{RI} / \mathrm{SMOM}$ as the intermediate schemes respectively.

\begin{tabular}{lcc}
\hline \hline Source & $\begin{array}{c}\text { Error } \\
(\%, \mathrm{RI} / \mathrm{MOM})\end{array}$ & $\begin{array}{c}\text { Error } \\
(\%, \mathrm{RI} / \mathrm{SMOM})\end{array}$ \\
\hline Statistical & 0.2 & 0.1 \\
Conversion ratio & 0.2 & 0.7 \\
$\Lambda_{\mathrm{QCD}}^{\mathrm{MS}}$ & 0.1 & 0.1 \\
Perturbative running & 0.2 & 0.2 \\
Lattice spacing & 0.2 & 0.1 \\
Fit range of $a^{2} p^{2}$ & 0.2 & 0.2 \\
$m_{s}^{\text {sea }} \neq 0$ & 1.9 & 0.2 \\
Total systematic uncertainty & 1.9 & 0.8 \\
\hline \hline
\end{tabular}

flat, from which we obtain a small uncertainty $0.2 \%$ as listed in Table IV. In the analyses below for $Z_{T}, Z_{S}$ and $Z_{P}$ obtained through the RI/SMOM scheme, we follow this method for estimating this uncertainty.

Adding the statistical and systematic uncertainties quadratically, we obtain $Z_{q}^{\overline{\mathrm{MS}}} / Z_{A}(2 \mathrm{GeV})=1.103(21)$ and 1.084(9) respectively for using the RI/MOM and $\mathrm{RI} / \mathrm{SMOM}$ as the intermediate schemes. These two numbers agree with each other within $1 \sigma$. Taking $1.103(21)$ as our final result and using the value $Z_{A}^{\mathrm{WI}}=1.1025(16)$ from Sec. III A, we find $Z_{q}^{\overline{\mathrm{MS}}}(2 \mathrm{GeV})=1.216(23)$ where the error includes the uncertainty propagated from $Z_{A}^{\mathrm{WI}}$.

\section{The tensor operator}

From Eq. (5) the ratio $Z_{T}^{\mathrm{RI} / \mathrm{MOM}} / Z_{A}^{\mathrm{WI}}=Z_{T}^{\mathrm{RI} / \mathrm{MOM}} / Z_{A}^{\mathrm{RI} / \mathrm{MOM}}$ at a given valence quark mass is computed by

$$
\frac{Z_{T}^{\mathrm{RI} / \mathrm{MOM}}}{Z_{A}^{\mathrm{RI} / \mathrm{MOM}}}=\left.\frac{\Gamma_{A}(p)}{\Gamma_{T}(p)}\right|_{p^{2}=\mu^{2}},
$$

where

$$
\Gamma_{T}(p)=\frac{1}{144} \operatorname{Tr}\left[\Lambda_{T, B}^{\mu \nu}(p) \sigma_{\mu \nu}\right]
$$

In the RI/SMOM scheme $Z_{T}^{\mathrm{RI} / \mathrm{SMOM}} / Z_{A}^{\mathrm{WI}}\left(=Z_{T}^{\mathrm{RI} / \mathrm{SMOM}} /\right.$ $\left.Z_{A}^{\mathrm{RI} / \mathrm{SMOM}}\right)$ at a given valence quark mass is obtained by using

$$
\frac{Z_{T}^{\mathrm{RI} / \mathrm{SMOM}}}{Z_{A}^{\mathrm{RI} / \mathrm{SMOM}}}=\left.\frac{\Gamma_{A}\left(p_{1}, p_{2}\right)}{\Gamma_{T}\left(p_{1}, p_{2}\right)}\right|_{\mathrm{sym}},
$$

where

$$
\Gamma_{T}\left(p_{1}, p_{2}\right)=\frac{1}{144} \operatorname{Tr}\left[\Lambda_{T, B}^{\mu \nu}\left(p_{1}, p_{2}\right) \sigma_{\mu \nu}\right]
$$



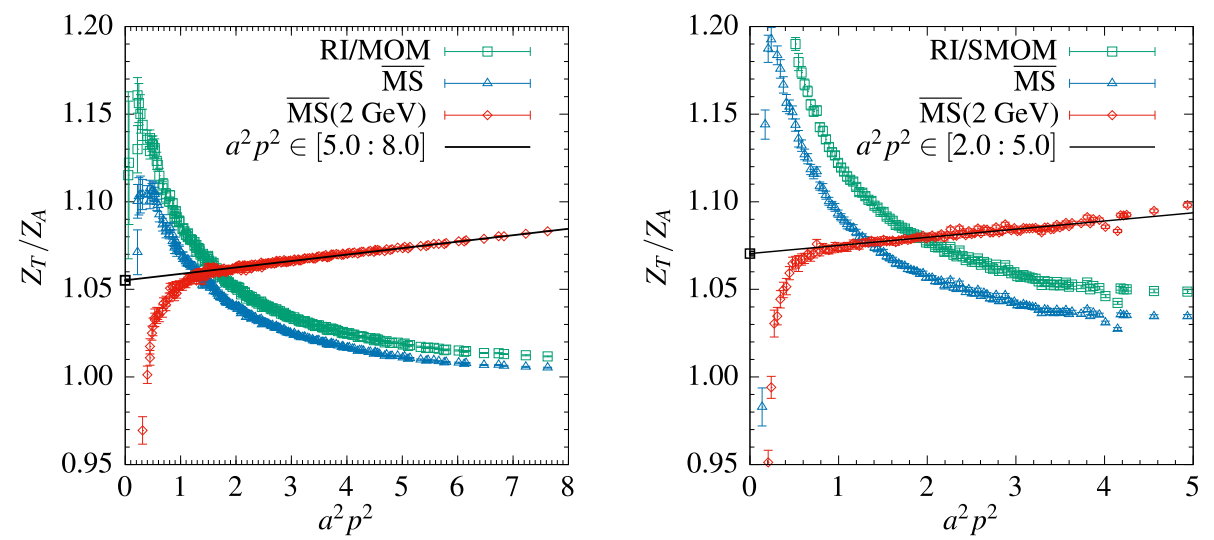

FIG. 8. Conversion of $Z_{T} / Z_{A}$ in the RI/MOM or RI/SMOM scheme to the $\overline{\mathrm{MS}}$ scheme. The running to $2 \mathrm{GeV}$ in the $\overline{\mathrm{MS}}$ scheme is shown by the red diamonds.

The numerical results of both $Z_{T}^{\mathrm{RI} / \mathrm{MOM}} / Z_{A}^{\mathrm{RI} / \mathrm{MOM}}$ and $Z_{T}^{\mathrm{RI} / \mathrm{SMOM}} / Z_{A}^{\mathrm{RI} / \mathrm{SMOM}}$ show little valence quark mass dependence. We perform linear extrapolations in $a m_{q}$ to get their chiral limit values, which are shown by the green squares in Fig. 8 as functions of the renormalization scale $a^{2} p^{2}$.

After the "democratic" cut in Eq. (18) on the momenta, the RI/MOM scheme results show a reasonable smooth behavior in $a^{2} p^{2}$. While some zigzag behavior can be seen in the RI/SMOM scheme results, which is from lattice discretization effects proportional to $a^{2} p^{[4]} / p^{2}$. For the RI/SMOM scheme, no "democratic" cut on the momenta $p_{1}, p_{2}$ and $q$ is applied since the symmetric conditions in Eq. (8) are not very easy to satisfy. This leads to the zigzag behavior (especially at large $a^{2} p^{2}$ ) reflecting lattice artifacts which are not $O(4)$ invariant.

If we start with the $Z_{q}^{\mathrm{RI} / \mathrm{SMOM}}$ defined in Eq. (11), then $Z_{T}^{\mathrm{RI} / \mathrm{SMOM}}$ is given by

$Z_{T}^{\mathrm{RI} / \mathrm{SMOM}}=\frac{Z_{q}^{\mathrm{RI} / \mathrm{SMOM}}}{\left.\Gamma_{T}\left(p_{1}, p_{2}\right)\right|_{\mathrm{sym}}}=\frac{\operatorname{Tr}\left[S_{B}^{-1}(p) \not p\right]_{p^{2}=\mu^{2}}}{\left.12 p^{2} \Gamma_{T}\left(p_{1}, p_{2}\right)\right|_{\mathrm{sym}}}$.

In practice we find that the $Z_{T}^{\mathrm{RI} / \mathrm{SMOM}}$ calculated in this way are even more scattered around a curve in $a^{2} p^{2}$, indicating larger discretization effects than in $Z_{T}^{\mathrm{RI} / \mathrm{SMOM}} / Z_{A}^{\mathrm{RI} / \mathrm{SMOM}}$. In the calculation of the ratio equation (34), these $O(4)$ noninvariant effects in the vertex functions in the denominator and numerator partially cancel. The zigzag behavior in the ratio is then less severe. Thus we always analyze the ratios of other RCs to $Z_{A}$ and in the end input the value of $Z_{A}^{\mathrm{WI}}$ to obtain their final results.

The perturbative conversion ratio for $Z_{T}^{\mathrm{RI} / \mathrm{MOM}}$ to the $\overline{\mathrm{MS}}$ scheme can be obtained from the conversion ratio for $Z_{T}^{\mathrm{RI}^{\prime} / \mathrm{MOM}}$ to $\overline{\mathrm{MS}}$, which was given in Ref. [24] to three loops, and $Z_{q}^{\mathrm{RI} / \mathrm{MOM}} / Z_{q}^{\mathrm{RI} / \mathrm{MOM}}$, whose three loop result can be computed by using the ratios $Z_{q}^{\mathrm{RI} / \mathrm{MOM}} / Z_{q}^{\overline{\mathrm{MS}}}$ and $Z_{q}^{\mathrm{RI} / \mathrm{MOM}} / Z_{q}^{\overline{\mathrm{MS}}}$ given in Ref. [19]. Thus we have

$$
\begin{aligned}
\frac{Z_{T}^{\overline{\mathrm{MS}}}}{Z_{T}^{\mathrm{RI} / \mathrm{MOM}}}= & \frac{Z_{T}^{\overline{\mathrm{MS}}}}{Z_{T}^{\mathrm{RI} / \mathrm{MOM}}} \frac{Z_{T}^{\mathrm{RI}} / \mathrm{MOM}}{Z_{T}^{\mathrm{RI} / \mathrm{MOM}}}=\frac{Z_{T}^{\overline{\mathrm{MS}}}}{Z_{T}^{\mathrm{RI} / \mathrm{MOM}}} \frac{Z_{q}^{\mathrm{RI}} / \mathrm{MOM}}{Z_{q}^{\mathrm{RI} / \mathrm{MOM}}} \\
= & 1-\frac{1}{81}\left(4866-1656 \xi_{3}-259 n_{f}\right)\left(\frac{\alpha_{s}}{4 \pi}\right)^{2} \\
& +\frac{2}{2187}\left(311424-105984 \xi_{3}-16576 n_{f}\right) \\
& \times\left(\frac{\alpha_{s}}{4 \pi}\right)^{3}+\mathcal{O}\left(\alpha_{s}^{4}\right) .
\end{aligned}
$$

At $p^{2}=\mu^{2}=16 \mathrm{GeV}^{2}$ and with three flavors of dynamical fermions, the numerical value of this ratio is

$$
\begin{aligned}
& \quad \frac{Z_{T}^{\overline{\mathrm{MS}}}}{Z_{T}^{\mathrm{R} / \mathrm{MOM}}}\left(\mu=4 \mathrm{GeV}, n_{f}=3\right) \\
& \quad=1+0.0 \alpha_{s}-0.1641 \alpha_{s}^{2}+0.0619 \alpha_{s}^{3}+\cdots \\
& \quad=1-0.0-0.0079+0.0006+\cdots
\end{aligned}
$$

The $\mathcal{O}\left(\alpha_{s}^{4}\right)$ term is around 0.0004 if assuming the coefficient of this term is the same as the one of the $\mathcal{O}\left(\alpha_{s}^{2}\right)$ term. Thus the truncation error in the conversion ratio is less than $0.1 \%$.

The two loop matching factor for converting $Z_{T}^{\mathrm{RI} / \mathrm{SMOM}}$ to the $\overline{\mathrm{MS}}$ scheme is given in Refs. [14,25]. In Landau gauge it reads

$$
\begin{aligned}
\frac{Z_{T}^{\overline{\mathrm{MS}}}}{Z_{T}^{\mathrm{RI} / \mathrm{SMOM}}=} & 1-0.21517295\left(\frac{\alpha_{s}}{4 \pi}\right) \\
& -\left(43.38395007-4.10327859 n_{f}\right)\left(\frac{\alpha_{s}}{4 \pi}\right)^{2} \\
& +\mathcal{O}\left(\alpha_{s}^{3}\right) .
\end{aligned}
$$

For $n_{f}=3$ and at $\mu=4 \mathrm{GeV}$, the above is 


$$
\begin{aligned}
& \quad \frac{Z_{T}^{\overline{\mathrm{MS}}}}{Z_{T}^{\mathrm{RI} / \mathrm{SMOM}}}\left(\mu=4 \mathrm{GeV}, n_{f}=3\right) \\
& \quad=1-0.017123 \alpha_{s}-0.196779 \alpha_{s}^{2}+\mathcal{O}\left(\alpha_{s}^{3}\right) \\
& \quad=1-0.0037-0.0094+\mathcal{O}\left(\alpha_{s}^{3}\right) .
\end{aligned}
$$

The two loop contribution is larger than the one loop contribution and is of size $\sim 1 \%$. At $a^{2} p^{2}=2$ (or $p=2.447 \mathrm{GeV}$ ) the suppression from $\alpha_{s}$ is even smaller $\left(\alpha_{s}^{\overline{\mathrm{MS}}}(2.447 \mathrm{GeV})=0.2678\right)$. To be conservative, we assign a $2 \%$ truncation error to $Z_{T}^{\overline{\mathrm{MS}}} / Z_{T}^{\mathrm{RI} / \mathrm{SMOM}}$ at the scale $p=2.447 \mathrm{GeV}$. Therefore for $Z_{T}^{\overline{\mathrm{MS}}}$ the conversion uncertainty in using RI/SMOM as the intermediate scheme seems to be much larger than that in using the RI/MOM scheme. It will be interesting to really calculate the three loop contribution for this conversion ratio.

The blue triangles in both graphs of Fig. 8 show the ratio $Z_{T}^{\overline{\mathrm{MS}}} / Z_{A}$ as a function of the renormalization scale $a^{2} p^{2}$. Their running to $2 \mathrm{GeV}$ is shown by the red diamonds, which are obtained by using the anomalous dimension $\gamma_{T}^{\overline{\mathrm{MS}}}$ in Landau gauge calculated up to and including four loops. We see a good linear dependence on $a^{2} p^{2}$ in $Z_{T}^{\overline{\mathrm{MS}}} / Z_{A}\left(2 \mathrm{GeV} ; a^{2} p^{2}\right)$ at large $a^{2} p^{2}$. Linear extrapolations in $a^{2} p^{2}$ in the range $a^{2} p^{2}>5$ and $a^{2} p^{2}>2$ are done respectively for the results from the two intermediate schemes RI/MOM and RI/SMOM. We find $Z_{T}^{\overline{\mathrm{MS}}} / Z_{A}(2 \mathrm{GeV})=1.0552(5)$ and $1.0704(12)$ respectively. The ranges are varied to estimate the associated systematic uncertainties, which are collected in Table V.

Similarly to the analyses of the other systematic uncertainties for $Z_{q}^{\overline{\mathrm{MS}}} / Z_{A}(2 \mathrm{GeV})$ in Sec. III C, we obtain the error budget for $Z_{T}^{\overline{\mathrm{MS}}} / Z_{A}(2 \mathrm{GeV})$ in Table V. The exception is the estimate of the sea quark mass dependence when using RI/MOM as the intermediate scheme. For the tensor current, we did not calculate its RC on the 24I ensemble. Thus we cannot combine the results on the 48I and 24I ensembles to estimate the error due to not being at the sea quark massless limit. We average the errors due to $m_{s}^{\text {sea }} \neq 0$

TABLE V. Uncertainties of $Z_{T}^{\overline{\mathrm{MS}}} / Z_{A}(2 \mathrm{GeV})$ in the chiral limit.

\begin{tabular}{lcc}
\hline \hline Source & $\begin{array}{c}\text { Error } \\
(\%, \mathrm{RI} / \mathrm{MOM})\end{array}$ & $\begin{array}{c}\text { Error } \\
(\%, \mathrm{RI} / \mathrm{SMOM})\end{array}$ \\
\hline Statistical & 0.05 & 0.1 \\
Conversion ratio & 0.1 & 2 \\
$\Lambda_{\mathrm{QCD}}^{\mathrm{MS}}$ & 0.1 & 0.1 \\
Perturbative running & $<0.01$ & $<0.01$ \\
Lattice spacing & 0.02 & 0.03 \\
Fit range of $a^{2} p^{2}$ & 0.1 & 0.1 \\
$m_{s}^{\text {sea }} \neq 0$ & 2.9 & 0.2 \\
Total systematic uncertainty & 2.9 & 2 \\
\hline \hline
\end{tabular}

TABLE VI. Uncertainties of $Z_{S}^{\overline{\mathrm{MS}}} / Z_{A}(2 \mathrm{GeV})$ and $Z_{P}^{\overline{\mathrm{MS}}} /$ $Z_{A}(2 \mathrm{GeV})$ in the chiral limit through the RI/MOM scheme.

\begin{tabular}{lcc}
\hline \hline Source & $\begin{array}{c}Z_{S}^{\overline{\mathrm{MS}}} / Z_{A} \\
(2 \mathrm{GeV})(\%)\end{array}$ & $\begin{array}{c}Z_{P}^{\overline{\mathrm{MS}}} / Z_{A} \\
(2 \mathrm{GeV})(\%)\end{array}$ \\
\hline Statistical & 0.1 & 0.6 \\
Conversion ratio & 1.5 & 1.5 \\
$\Lambda_{\mathrm{QCD}}^{\overline{\mathrm{MS}}}$ & 0.3 & 0.4 \\
Perturbative running & 0.1 & 0.1 \\
Lattice spacing & 0.1 & $<0.1$ \\
Fit range of $a^{2} p^{2}$ & 0.1 & 0.3 \\
Finite-volume effects & $\ldots$ & 0.3 \\
$m_{s}^{\text {sea }} \neq 0$ & 2.1 & 4.7 \\
Total systematic uncertainty & 2.6 & 5.0 \\
\hline \hline
\end{tabular}

for the RCs of the quark field, the scalar and pseudoscalar densities in Table IV and Table VI and treat the average as the corresponding error estimate for $Z_{T}^{\overline{\mathrm{MS}}} / Z_{A}(2 \mathrm{GeV})$. The light sea quark mass dependence is neglected since the light sea quark mass is very close to the chiral limit on the 48I ensemble.

Adding the statistical and systematic uncertainties quadratically, we get $Z_{T}^{\overline{\mathrm{MS}}} / Z_{A}(2 \mathrm{GeV})=1.055(31)$ and $1.070(21)$ respectively for using the RI/MOM and RI/ SMOM as the intermediate schemes. These two numbers are in agreement within $1 \sigma$. The result from the RI/SMOM scheme has a large systematic error from the conversion ratio. While the result from the RI/MOM scheme has a dominant error from the strange sea quark mass dependence. Taking 1.055(31) as our final result and using the value $Z_{A}^{\mathrm{WI}}=1.1025(16)$ from Sec. III A, we get $Z_{T}^{\overline{\mathrm{MS}}}(2 \mathrm{GeV})=1.163(34)$ where the error includes the uncertainty propagated from $Z_{A}^{\mathrm{WI}}$.

\section{E. The scalar density}

The calculation of $Z_{S}^{\mathrm{RI} / \mathrm{MOM}}$ and its conversion to the $\overline{\mathrm{MS}}$ scheme closely follow our previous work [17] on the $24^{3} \times 64 \mathrm{RBC} / \mathrm{UKQCD}$ lattices (24I) with similar lattice spacings. One difference is that now we can impose a stronger "democratic" cut [Eq. (18)] on the momentum modes since the lattice size is now bigger. This leads to a smoother dependence on the renormalization scale $a^{2} p^{2}$ in $Z_{S}^{\mathrm{RI} / \mathrm{MOM}}$ since the Lorentz noninvariant lattice artifacts are further reduced. Another difference is that here we analyze the ratio $Z_{S}^{\mathrm{RI} / \mathrm{MOM}} / Z_{A}^{\mathrm{RI} / \mathrm{MOM}}=\Gamma_{A}(p) /\left.\Gamma_{S}(p)\right|_{p^{2}=\mu^{2}}$ instead of the absolute $Z_{S}^{\mathrm{RI} / \mathrm{MOM}}$.

The chiral extrapolation of $Z_{S}^{\mathrm{RI} / \mathrm{MOM}} / Z_{A}^{\mathrm{RI} / \mathrm{MOM}}$ is done with an Ansatz, with three parameters $A_{s}, B_{s}$ and $C_{s}[11,17,26]$

$$
Z_{S} / Z_{A}=\frac{A_{s}}{\left(a m_{q}\right)^{2}}+B_{s}+C_{s} \cdot a m_{q},
$$



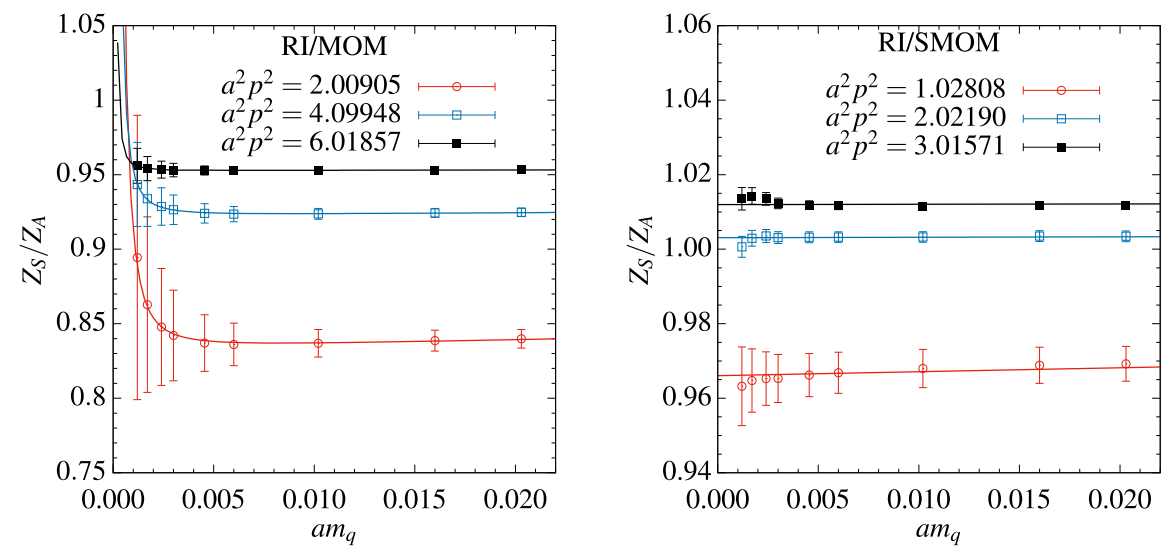

FIG. 9. Chiral extrapolations of $Z_{S} / Z_{A}$ in the RI/MOM scheme using Eq. (41) (left panel) and in the RI/SMOM scheme using a linear function (right panel).

where the double pole term comes from the topological zero modes of the overlap fermions. $B_{s}$ is taken as the chiral limit value of $Z_{S}^{\mathrm{RI} / \mathrm{MOM}} / Z_{A}^{\mathrm{RI} / \mathrm{MOM}}$. Examples of this extrapolation are shown in the left panel of Fig. 9.

The valence quark mass dependence of $Z_{S}^{\mathrm{RI} / \mathrm{SMOM} /}$ $Z_{A}^{\mathrm{RI} / \mathrm{SMOM}}$ seems to be milder, which is computed from the ratio $\Gamma_{A}\left(p_{1}, p_{2}\right) /\left.\Gamma_{S}\left(p_{1}, p_{2}\right)\right|_{\text {sym }}$. We tried both Eq. (41) and a linear function in quark mass for going to the chiral limit (only the linear extrapolation is shown in the right panel of Fig. 9). We find consistent chiral limit values from the two extrapolations for $Z_{S}^{\mathrm{RI} / \mathrm{SMOM}} / Z_{A}^{\mathrm{RI} / \mathrm{SMOM}}$.

The scale dependence of $Z_{S} / Z_{A}$ is shown in Fig. 10 by the green squares. The conversion to $\overline{\mathrm{MS}}$ and the running to $2 \mathrm{GeV}$ in $\overline{\mathrm{MS}}$ are shown in the same figure by the blue triangles and the red diamonds respectively. In the right panel the green squares and the blue triangles are much closer together than in the left panel. This is because the conversion ratio from the RI/SMOM scheme to the $\overline{\mathrm{MS}}$ scheme is much closer to 1 than the ratio from the RI/MOM scheme to $\overline{\mathrm{MS}}$. This leads to a much smaller systematic uncertainty from the conversion in using RI/SMOM as the intermediate scheme. As we have estimated in our previous work [17], the truncation error in the conversion ratio $Z_{S}^{\overline{\mathrm{MS}}} / Z_{S}^{\mathrm{RI} / \mathrm{MOM}}$ (three loop result) above $4 \mathrm{GeV}$ is around $1.5 \%$. The inverse of the conversion ratio $Z_{S}^{\overline{\mathrm{MS}}} / Z_{S}^{\mathrm{RI} / \mathrm{SMOM}}$ has been calculated up to two loops [13,14]

$$
\begin{aligned}
\frac{Z_{S}^{\mathrm{RI} / \mathrm{SMOM}}}{Z_{S}^{\overline{\mathrm{MS}}}}= & \frac{Z_{m}^{\overline{\mathrm{MS}}}}{Z_{m}^{\mathrm{RI} / \mathrm{SMOM}}} \\
= & 1-0.6455188560\left(\frac{\alpha_{s}}{4 \pi}\right) \\
& -\left(22.60768757-4.013539470 n_{f}\right)\left(\frac{\alpha_{s}}{4 \pi}\right)^{2} .
\end{aligned}
$$

From the above we find for $n_{f}=3$ and at $a^{2} p^{2}=2$ $(p=2.447 \mathrm{GeV})$

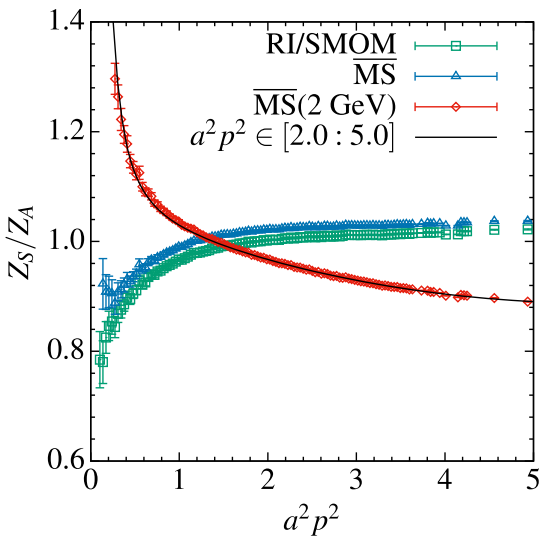

FIG. 10. Conversion and running of $Z_{S} / Z_{A}$ for the intermediate schemes RI/MOM (left) and RI/SMOM (right). The black curve in the right panel is a fitting using Ansatz (e) in Eq. (48). 


$$
\begin{gathered}
\frac{Z_{S}^{\overline{\mathrm{MS}}}}{Z_{S}^{\mathrm{RI} / \mathrm{SMOM}}}=1.0+0.051369 \alpha_{s}+0.069556 \alpha_{s}^{2}+\mathcal{O}\left(\alpha_{s}^{3}\right) \\
\stackrel{p=2.447}{=} \mathrm{GeV} 1.0+0.0138+0.0050+\mathcal{O}\left(\alpha_{s}^{3}\right) .
\end{gathered}
$$

Assuming the coefficient for the $\mathcal{O}\left(\alpha_{s}^{3}\right)$ term is $0.069556 \times(0.069556 / 0.051369)=0.094182$, we expect the $\mathcal{O}\left(\alpha_{s}^{3}\right)$ term to be of size $\sim 0.0018$. This means the truncation error can be estimated to be $0.2 \%$, which is much smaller than the $1.5 \%$ for the conversion of the RI/MOM scheme result.

After the running to $2 \mathrm{GeV}$ in the $\overline{\mathrm{MS}}$ scheme, $Z_{S}^{\overline{\mathrm{MS}}}\left(2 \mathrm{GeV} ; a^{2} p^{2}\right) / Z_{A}$ shows a good linear behavior in the initial scale $a^{2} p^{2}$ at large scales in using RI/MOM as the intermediate scheme. This is shown by the red diamonds in the left panel of Fig. 10. The nonzero slope of the red diamonds is attributed to lattice discretization effects proportional to $a^{2} p^{2}$. Above $a^{2} p^{2}=5$ we can do a linear extrapolation to $a^{2} p^{2}=0$ with good $\chi^{2} /$ d.o.f. and obtain $Z_{S}^{\overline{\mathrm{MS}}}(2 \mathrm{GeV}) / Z_{A}=1.0137(13)$. Varying the extrapolation range to $a^{2} p^{2}>4$, we get $Z_{S}^{\overline{\mathrm{MS}}}(2 \mathrm{GeV}) / Z_{A}=1.0152(8)$. The variation in the center value $(0.1 \%)$ is taken as one of the systematic uncertainties.

The statistical and systematic uncertainties of $Z_{S}^{\overline{\mathrm{MS}}} /$ $Z_{A}(2 \mathrm{GeV})$ obtained by using RI/MOM as the intermediate scheme are listed in Table VI. The uncertainties from the strange sea quark mass dependence and from the conversion ratio dominate. Adding all the uncertainties quadratically, we get $Z_{S}^{\overline{\mathrm{MS}}} / Z_{A}(2 \mathrm{GeV})=1.014(26)$. Using the value $Z_{A}^{\mathrm{WI}}=1.1025(16)$ from Sec. III A, we find $Z_{S}^{\overline{\mathrm{MS}}}(2 \mathrm{GeV})=1.118(29)$ where the error includes the uncertainty propagated from $Z_{A}^{\mathrm{WI}}$. This number agrees with our previous result $Z_{S}^{\overline{\mathrm{MS}}}(2 \mathrm{GeV})=1.127(21)$ on the $24^{3} \times 64$ lattice [17].

In the right graph of Fig. $10, Z_{S}^{\overline{\mathrm{MS}}}\left(2 \mathrm{GeV} ; a^{2} p^{2}\right) / Z_{A}$ obtained by using RI/SMOM as the intermediate scheme is shown as a function of the initial scale $a^{2} p^{2}$. We do not see a clear window, in which $Z_{S}^{\overline{\mathrm{MS}}}\left(2 \mathrm{GeV} ; a^{2} p^{2}\right) / Z_{A}$ linearly depends on $a^{2} p^{2}$. This is quite different from what we saw in $Z_{q}^{\overline{\mathrm{MS}}}\left(2 \mathrm{GeV} ; a^{2} p^{2}\right) / Z_{A}$ and $Z_{T}^{\overline{\mathrm{MS}}}\left(2 \mathrm{GeV} ; a^{2} p^{2}\right) / Z_{A}$ (the right panels in Figs. 7 and 8 respectively), which are also obtained through the RI/SMOM scheme. To model the behavior of $Z_{S}^{\overline{\mathrm{MS}}}\left(2 \mathrm{GeV} ; a^{2} p^{2}\right) / Z_{A}$, we tried several Ansätze (with $x \equiv a^{2} p^{2}$ and fitting parameters $A, B, C$, $D$ and $E)$ :

$$
\begin{aligned}
& (a): f(x)=A+B x+C x^{2}, \\
& (b): f(x)=A+B x+D / x, \\
& (c): f(x)=A+B x+E / x^{2}, \\
& (d): f(x)=A+B x+C x^{2}+D / x, \\
& (e): f(x)=A+B x+C x^{2}+E / x^{2},
\end{aligned}
$$

where the $C\left(a^{2} p^{2}\right)^{2}$ term is for higher order discretization effects and the $1 / x^{n}(n=1,2)$ terms are for possible nonperturbative effects. In using the above Ansätze to fit our data, we fix the upper limit of $a^{2} p^{2}$ to 5 and vary the lower limit. We collect the $\chi^{2} /$ d.o.f. and the results of $A$ for various fitting ranges in Table VII. We find the $C\left(a^{2} p^{2}\right)^{2}$ term is necessary to decrease the $\chi^{2} /$ d.o.f. Models $(a),(d)$ and $(e)$ give smaller $\chi^{2} /$ d.o.f. than models $(b)$ and $(c)$ in almost all fitting ranges [the only exception case is with $\left(a^{2} p^{2}\right)_{\min }=0.5$, in which model $(a)$ gives a larger $\chi^{2} /$ d.o.f. than model $\left.(b)\right]$. For the behavior of the possible nonperturbative effects, the data can hardly distinguish between $1 / x$ and $1 / x^{2}$ [the $\chi^{2} /$ d.o.f. of models $(d)$ and $(e)$

\begin{tabular}{|c|c|c|c|c|c|c|c|c|c|c|}
\hline \multirow[b]{2}{*}{$x_{\min }$} & \multicolumn{2}{|c|}{ (a) } & \multicolumn{2}{|c|}{ (b) } & \multicolumn{2}{|c|}{ (c) } & \multicolumn{2}{|c|}{ (d) } & \multicolumn{2}{|c|}{ (e) } \\
\hline & $A$ & $\chi^{2} /$ d.o.f. & $A$ & $\chi^{2} /$ d.o.f. & $A$ & $\chi^{2} /$ d.o.f. & $A$ & $\chi^{2} /$ d.o.f. & $A$ & $\chi^{2} /$ d.o.f. \\
\hline 0.5 & $1.096(2)$ & 8.56 & $0.941(3)$ & 7.83 & $1.000(2)$ & 20.2 & $1.015(6)$ & 2.95 & $1.064(3)$ & 3.01 \\
\hline 1.0 & $1.091(2)$ & 5.37 & $0.930(4)$ & 6.79 & $0.986(2)$ & 12.2 & $1.020(10)$ & 3.41 & $1.057(5)$ & 3.41 \\
\hline 1.5 & $1.084(2)$ & 4.52 & $0.915(5)$ & 6.10 & $0.973(3)$ & 8.57 & $1.027(19)$ & 4.03 & $1.056(9)$ & 4.02 \\
\hline 1.8 & $1.080(3)$ & 4.16 & $0.904(6)$ & 6.07 & $0.964(3)$ & 7.58 & $1.040(30)$ & 4.54 & $1.060(15)$ & 4.54 \\
\hline 2.0 & $1.078(3)$ & 4.92 & $0.895(7)$ & 6.13 & $0.957(4)$ & 7.19 & $1.050(42)$ & 4.97 & $1.064(21)$ & 4.97 \\
\hline 2.2 & $1.077(4)$ & 5.39 & $0.883(9)$ & 6.18 & $0.948(4)$ & 5.47 & $1.046(61)$ & 5.47 & $1.061(30)$ & 5.47 \\
\hline 2.5 & $1.074(6)$ & 5.99 & $0.869(14)$ & 6.63 & $0.938(7)$ & 7.04 & $1.08(10)$ & 6.14 & $1.078(53)$ & 6.14 \\
\hline 2.8 & $1.074(10)$ & 6.69 & $0.843(22)$ & 6.98 & $0.921(12)$ & 7.18 & $1.08(20)$ & 6.89 & $1.08(10)$ & 6.89 \\
\hline 3.0 & $1.067(15)$ & 7.41 & $0.834(33)$ & 7.68 & $0.913(17)$ & 7.81 & $1.17(33)$ & 7.66 & $1.12(17)$ & 7.66 \\
\hline
\end{tabular}
are almost all the same for all fitting ranges]. Above $\left(a^{2} p^{2}\right)_{\min }=\sim 1.5$, the possible nonperturbative effects $\left(1 / x^{n}\right.$ terms) can be ignored: Model (a) gives comparable $\chi^{2} /$ d.o.f. as or smaller $\chi^{2} /$ d.o.f. than models $(d)$ and $(e)$.

TABLE VII. Fittings of $Z_{S}^{\overline{\mathrm{MS}}} / Z_{A}\left(2 \mathrm{GeV} ; a^{2} p^{2}\right)$ obtained from the RI/SMOM scheme to the models in Eqs. (44)-(48). Here we vary the lower limit of $x\left(\equiv a^{2} p^{2}\right)$. The uncertainties have been inflated by the factor $\sqrt{\chi^{2} / \text { d.o.f. }}$ 


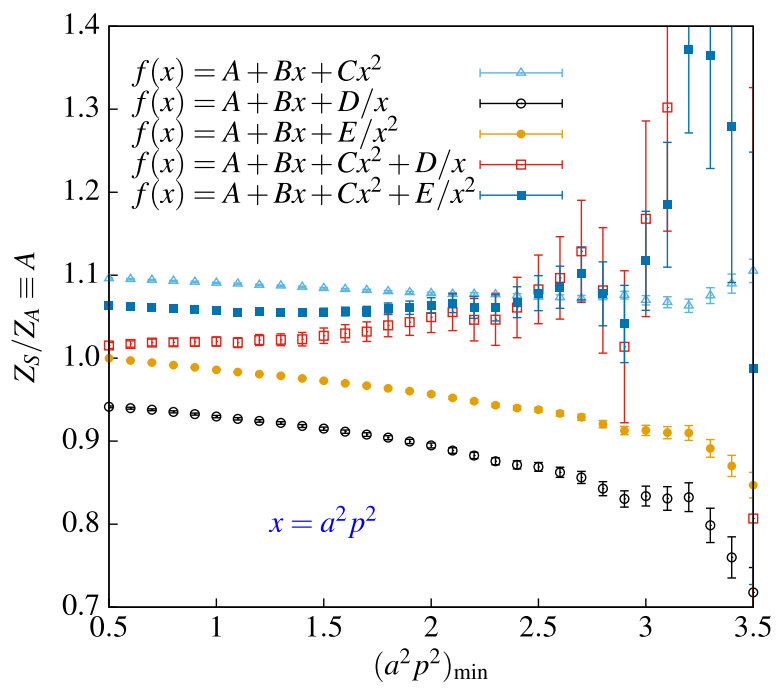

FIG. 11. $Z_{S}^{\overline{\mathrm{MS}}} / Z_{A}(2 \mathrm{GeV})$ from the RI/SMOM scheme by fitting $Z_{S}^{\overline{\mathrm{MS}}} / Z_{A}\left(2 \mathrm{GeV} ; a^{2} p^{2}\right)$ to the five Ansätze equations (44)-(48) as we vary the lower limit $\left(a^{2} p^{2}\right)_{\min }$ of the fitting range $\left[\left(a^{2} p^{2}\right)_{\min }, 5\right]$.

Also the fitted parameter $D(E)$ in model $(d)[(e)]$ is consistent with zero within its uncertainty. This is of course expected since the nonperturbative effects are suppressed at large momentum scale.

The resulted $A$ 's from all fittings are plotted in Fig. 11 as functions of the lower limit $\left(a^{2} p^{2}\right)_{\min }$. Above $\left(a^{2} p^{2}\right)_{\min }=\sim 2$, the results from models $(a),(d)$ and $(e)$ converge to a more or less stable value. In the range $\left(a^{2} p^{2}\right)_{\min } \in[2,3]$ the three models give consistent results. Taking $\left(a^{2} p^{2}\right)_{\min }=2$ and averaging the $A$ 's from the three models, we find $Z_{S}^{\overline{\mathrm{MS}}} / Z_{A}(2 \mathrm{GeV})=1.064(42)$. Here the statistical error is the biggest of the three ones from the three fittings. The span $(0.028$ or $2.6 \%)$ in the three center values will be taken as one source of the systematic uncertainties.

In Table VIII we collect our error analyses for $Z_{S}^{\overline{\mathrm{MS}}} / Z_{A}(2 \mathrm{GeV})$ obtained through the RI/SMOM scheme. In total, we find a $5.1 \%$ error in $Z_{S}^{\overline{\mathrm{MS}}} / Z_{A}(2 \mathrm{GeV})$. That is to say, $Z_{S}^{\overline{\mathrm{MS}}} / Z_{A}(2 \mathrm{GeV})=1.064(55)$. This result, with a relatively large error, agrees with $Z_{S}^{\overline{\mathrm{MS}}} / Z_{A}(2 \mathrm{GeV})=$ 1.014(26) obtained by using the RI/MOM scheme. The fact that we cannot get a broad window of $a^{2} p^{2}$ in $Z_{S}^{\overline{\mathrm{MS}}} / Z_{A}\left(2 \mathrm{GeV} ; a^{2} p^{2}\right)$, in which both the nonperturbative effects and the lattice discretization effects are small, leads to the large uncertainty in $Z_{S}^{\overline{\mathrm{MS}}} / Z_{A}(2 \mathrm{GeV})$ when using the RI/SMOM scheme. This may come from the HYP smearing that we do on the gauge fields. In Ref. [27] the upper edge of the renormalization window was found to be reduced by link smearing. We may do a calculation on thin link configurations to check this in the future.
TABLE VIII. Uncertainties of $Z_{S}^{\overline{\mathrm{MS}}} / Z_{A}(2 \mathrm{GeV})$ and $Z_{P}^{\overline{\mathrm{MS}}} /$ $Z_{A}(2 \mathrm{GeV})$ in the chiral limit through the RI/SMOM scheme.

\begin{tabular}{lcc}
\hline \hline Source & $\begin{array}{c}Z_{S}^{\overline{\mathrm{MS}}} / Z_{A} \\
(2 \mathrm{GeV})(\%)\end{array}$ & $\begin{array}{c}Z_{P}^{\overline{\mathrm{MS}}} / Z_{A} \\
(2 \mathrm{GeV})(\%)\end{array}$ \\
\hline Statistical & 3.9 & 4.4 \\
Conversion ratio & 0.2 & 0.2 \\
$\Lambda_{\mathrm{QCD}}^{\overline{\mathrm{MS}}}$ & 0.2 & 0.1 \\
Perturbative running & 0.1 & 0.1 \\
Lattice spacing & 0.1 & 0.1 \\
Fit range of $a^{2} p^{2}$ & 2.1 & 1.2 \\
Span in the results from models & 2.6 & 3.0 \\
$\quad(a),(d) \&(e)$ & & 0.2 \\
$m_{s}^{\text {sea }} \neq 0$ & 0.2 & 3.2 \\
Total systematic uncertainty & 3.4 & \\
\hline \hline
\end{tabular}

\section{F. The pseudoscalar density}

For the calculation of $Z_{P}^{\mathrm{RI} / \mathrm{MOM}} / Z_{A}$ and its conversion to $\overline{\mathrm{MS}}$, we again closely follow our previous work [17]. The Goldstone boson contamination in $Z_{P}^{\mathrm{RI} / \mathrm{MOM}} / Z_{A}$ is apparent as we can see from the left panel in Fig. 12. This contamination in the forward vertex function $\Gamma_{P}(p)$ is proportional to $1 /\left(m_{\pi}^{2} p^{2}\right)$ or $1 /\left(m_{q} p^{2}\right)$ due to the pion propagator. Thus the chiral extrapolation of $Z_{P}^{\mathrm{RI} / \mathrm{MOM}} / Z_{A}$ at a fixed $a^{2} p^{2}$ is done by using the Ansatz

$$
\left(Z_{P}^{\mathrm{RI} / \mathrm{MOM}} / Z_{A}\right)^{-1}=\frac{A_{p}}{a m_{q}}+B_{p}+C_{p} \cdot a m_{q},
$$

where $A_{p}, B_{p}$ and $C_{p}$ are three fitting parameters. $B_{p}^{-1}$ is taken as the chiral limit value for $Z_{P}^{\mathrm{RI} / \mathrm{MOM}} / Z_{A}$. Examples of this chiral extrapolation are shown in the left panel of Fig. 13. The data points at the three lightest masses sit well on the fitting curves at almost all $a^{2} p^{2}$ values (exceptions are the very small $a^{2} p^{2}$ 's). To estimate the possible finitevolume effects at small quark masses, in the following we will repeat our analyses without the data at the four lightest quark masses and take the change in $Z_{P}^{\overline{\mathrm{MS}}}(2 \mathrm{GeV}) / Z_{A}$ as one of its systematic uncertainties.

In the SMOM scheme, the Goldstone boson contamination in $\left.\Gamma_{P}\left(p_{1}, p_{2}\right)\right|_{\text {sym }}$ is proportional to $1 / q^{2}\left(=1 / p^{2}\right)$ since $m_{\pi}^{2} \ll q^{2}$. Thus the quark mass dependence of $Z_{P}^{\mathrm{RI} / \mathrm{SMOM}} / Z_{A}$ should be small provided $m_{q}^{2} \ll q^{2}$. This can be clearly seen in the right graph of Fig. 12 and also in the right panel of Fig. 13, in which we do linear chiral extrapolations for $Z_{P}^{\mathrm{RI} / \mathrm{SMOM}} / Z_{A}$ at three typical momentum values.

Figure 14 shows the ratio $Z_{P} / Z_{S}$ in the extrapolated chiral limit in both the RI/MOM and RI/SMOM schemes. These results verify the relation $Z_{S}=Z_{P}$ for overlap fermions. The RI/SMOM scheme suppresses the Goldstone boson contamination in the pseudoscalar vertex 

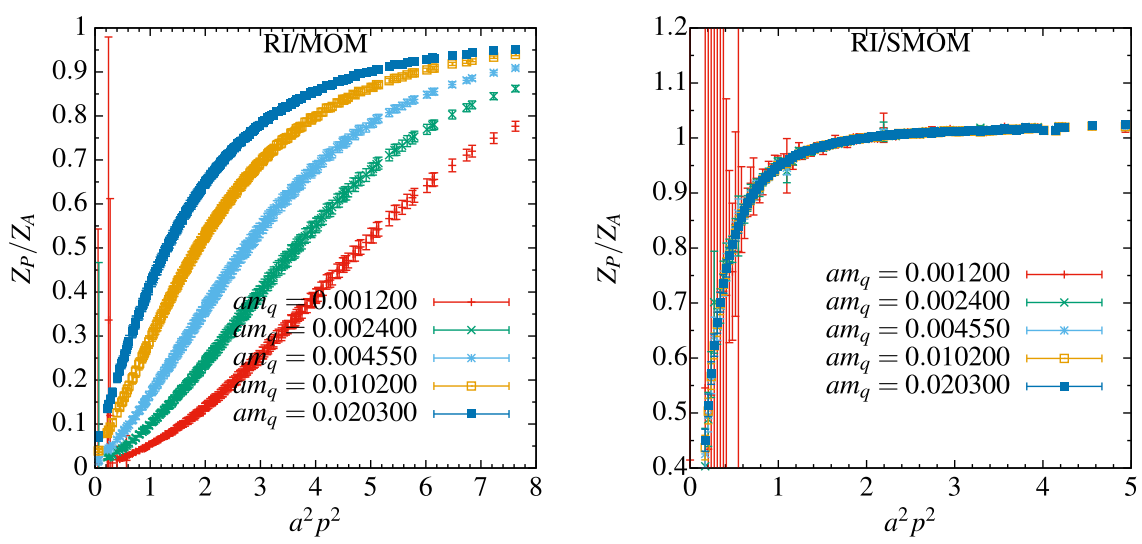

FIG. 12. $Z_{P} / Z_{A}$ in the schemes RI/MOM (left graph) and RI/SMOM (right graph).
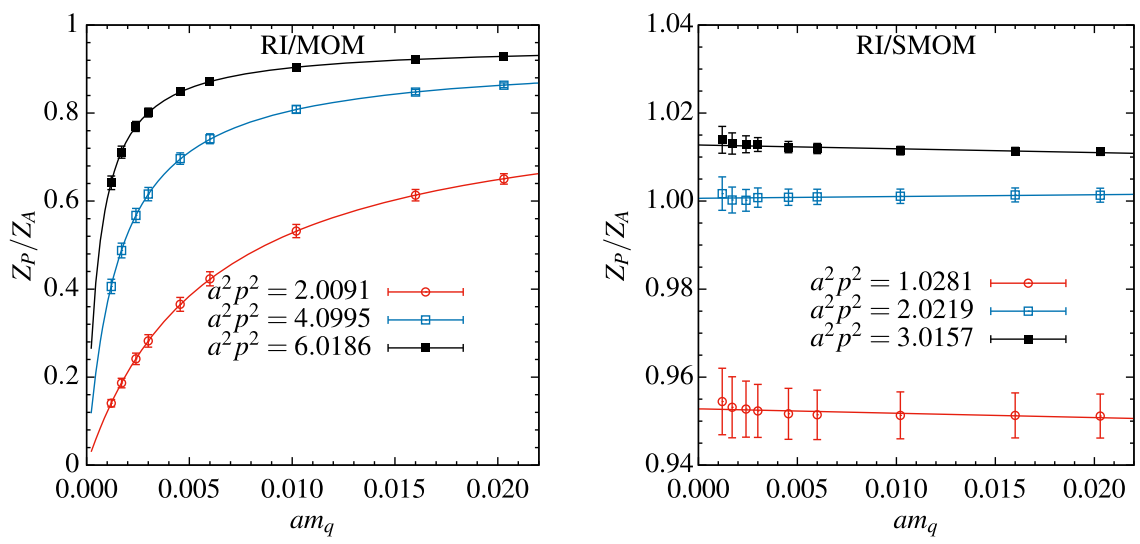

FIG. 13. Examples of chiral extrapolations of $Z_{P} / Z_{A}$ in the RI/MOM (left graph) and RI/SMOM (right graph) schemes.

function since $q^{2} \neq 0$. Thus $Z_{P} / Z_{S}=1$ is more precisely satisfied in this scheme than in the RI/MOM scheme.

The conversion to and running in the $\overline{\mathrm{MS}}$ scheme for both $Z_{P}^{\mathrm{RI} / \mathrm{MOM}} / Z_{A}$ and $Z_{P}^{\mathrm{RI} / \mathrm{SMOM}} / Z_{A}$ are shown in Fig. 15 .

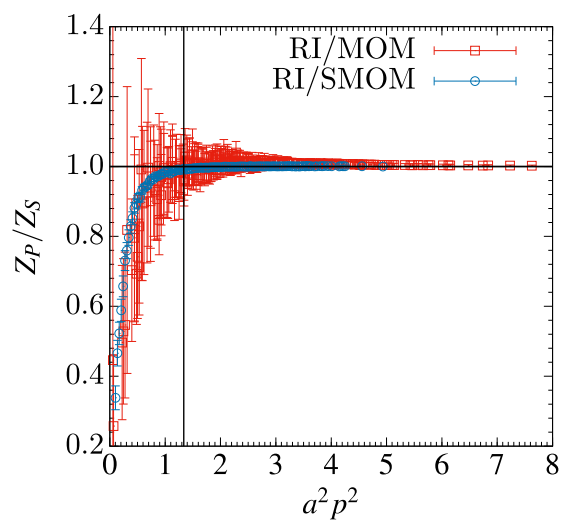

FIG. 14. $Z_{P} / Z_{S}$ in the extrapolated chiral limit in the RI/MOM and RI/SMOM schemes. $Z_{P} / Z_{S}=1$ is well satisfied in both schemes at large momentum scale. In the RI/MOM result the Goldstone boson contamination has been removed by using Eq. (49). The horizontal line is $Z_{P} / Z_{S}=1$ for guiding the eyes. The vertical line indicates $p=2 \mathrm{GeV}$.
The two conversion ratios used here are the same as those for $Z_{S}^{\mathrm{RI} / \mathrm{MOM}} / Z_{A}$ and $Z_{S}^{\mathrm{RI} / \mathrm{SMOM}} / Z_{A}$ respectively. From the $\mathrm{RI} / \mathrm{MOM}$ result, a linear extrapolation in $a^{2} p^{2}$ for $Z_{P}^{\overline{\mathrm{MS}}}\left(2 \mathrm{GeV} ; a^{2} p^{2}\right) / Z_{A}$ gives $Z_{P}^{\overline{\mathrm{MS}}}(2 \mathrm{GeV}) / Z_{A}=1.019(6)$. It agrees with $Z_{S}^{\overline{\mathrm{MS}}}(2 \mathrm{GeV}) / Z_{A}=1.0137(13)$ obtained in Sec. III E also by using RI/MOM as the intermediate scheme. We repeat our analyses dropping the data points at the four lightest quark masses and obtain $Z_{P}^{\overline{\mathrm{MS}}}(2 \mathrm{GeV}) / Z_{A}=1.022(9)$. It agrees with $1.019(6)$ within the uncertainty. The shift in the center value is taken as a systematic uncertainty from possible finitevolume effects. In the last column of Table VI we summarize our error analyses for $Z_{P}^{\overline{\mathrm{MS}}}(2 \mathrm{GeV}) / Z_{A}$. Our final result is $Z_{P}^{\overline{\mathrm{MS}}}(2 \mathrm{GeV}) / Z_{A}=1.019(6)(51)$, where the second error is the total systematic uncertainty. Using the value $Z_{A}^{\mathrm{WI}}=1.1025(16)$ from Sec. III A, we get $Z_{P}^{\overline{\mathrm{MS}}}(2 \mathrm{GeV})=1.123(56)$ where the error includes the uncertainty propagated from $Z_{A}^{\mathrm{WI}}$.

When we convert the RI/SMOM result $Z_{P}^{\mathrm{RI} / \mathrm{SMOM}}\left(a^{2} p^{2}\right) / Z_{A}$ into the $\overline{\mathrm{MS}}$ scheme and run it to $2 \mathrm{GeV}$, we find that the behavior of $Z_{P}^{\overline{\mathrm{MS}}}\left(2 \mathrm{GeV} ; a^{2} p^{2}\right) / Z_{A}$ 

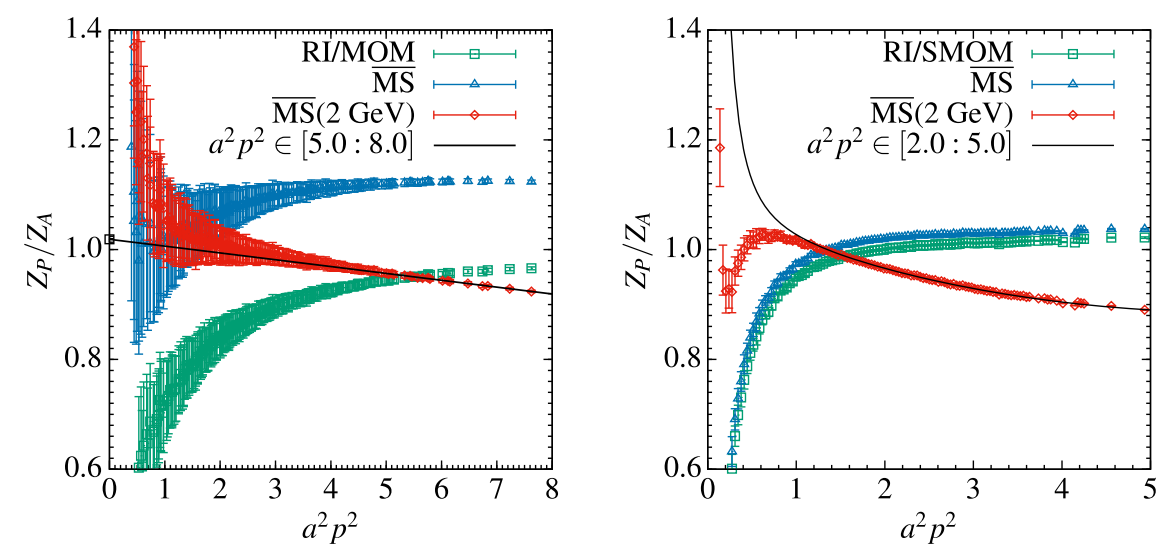

FIG. 15. The conversion and running of $Z_{P} / Z_{A}$ using RI/MOM (left) or RI/SMOM (right) as the intermediate scheme. The black curve in the right panel is a fitting using Ansatz (e) in Eq. (48).

(see the right panel in Fig. 15) looks similar to that of $Z_{S}^{\overline{\mathrm{MS}}}\left(2 \mathrm{GeV} ; a^{2} p^{2}\right) / Z_{A}$ in the right panel of Fig. 10 except at the very small $a^{2} p^{2}$ region. We therefore also tried the Ansätze in Eqs. (44)-(48) to remove the lattice discretization artifacts and possible nonperturbative effects in $Z_{P}^{\overline{\mathrm{MS}}}\left(2 \mathrm{GeV} ; a^{2} p^{2}\right) / Z_{A}$ to get $Z_{P}^{\overline{\mathrm{MS}}}(2 \mathrm{GeV}) / Z_{A}$. The fitting results are similar to those for $Z_{S}^{\overline{\mathrm{MS}}}\left(2 \mathrm{GeV} ; a^{2} p^{2}\right) / Z_{A}$ from the RI/SMOM scheme: The $C\left(a^{2} p^{2}\right)^{2}$ term is necessary to reduce the $\chi^{2} /$ d.o.f. Above $\left(a^{2} p^{2}\right)_{\min }=\sim 1.5$, the nonperturbative effects $\left(1 / x^{n}\right.$ terms) can be ignored.

A graph similar to Fig. 11 is obtained as shown in Fig. 16 for the fitted parameter $A=Z_{P}^{\overline{\mathrm{MS}}}(2 \mathrm{GeV}) / Z_{A}$. Averaging the results from models $(a),(d)$ and $(e)$ with $\left(a^{2} p^{2}\right)_{\min }=2$, we get $Z_{P}^{\overline{\mathrm{MS}}}(2 \mathrm{GeV}) / Z_{A}=1.056(47)$. Here again we take the biggest uncertainty from the three fits as our statistical

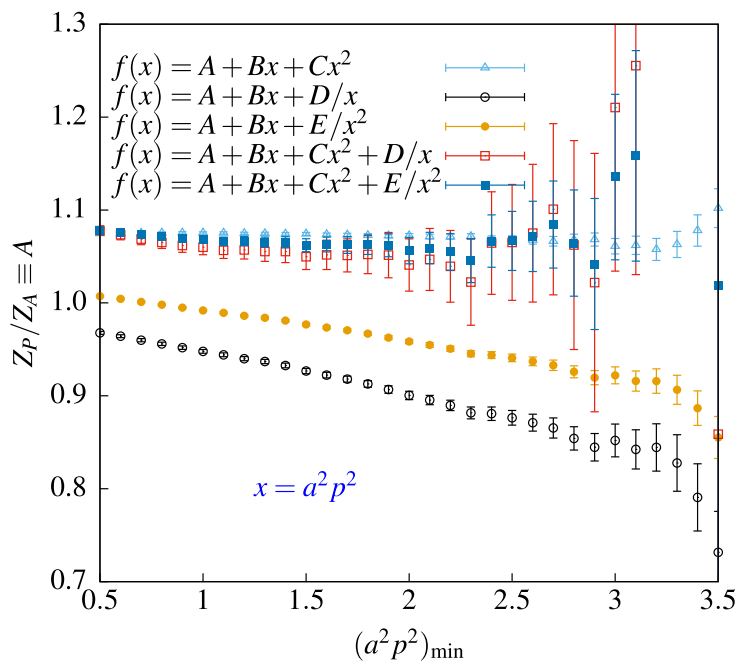

FIG. 16. Results of $A=Z_{P}^{\overline{\mathrm{MS}}}(2 \mathrm{GeV}) / Z_{A}$ from fitting $Z_{P}^{\overline{\mathrm{MS}}}\left(2 \mathrm{GeV} ; a^{2} p^{2}\right) / Z_{A}$ to the Ansätze in Eqs. (44)-(48) when using RI/SMOM as the intermediate scheme. The lower limit $\left(a^{2} p^{2}\right)_{\min }$ of the fitting range $\left[\left(a^{2} p^{2}\right)_{\min }, 5\right]$ is varied. error. The analyses of uncertainties for $Z_{P}^{\overline{\mathrm{MS}}}(2 \mathrm{GeV}) / Z_{A}$ are given in Table VIII. Adding all the uncertainties quadratically, we finally obtain $Z_{P}^{\overline{\mathrm{MS}}}(2 \mathrm{GeV}) / Z_{A}=$ 1.056(58). This number agrees with $Z_{S}^{\overline{\mathrm{MS}}}(2 \mathrm{GeV}) / Z_{A}=$ 1.064(55) from using also the RI/SMOM scheme.

\section{SUMMARY}

In this paper we present our calculation of the RCs for the quark field $\left(Z_{q}\right)$ and bilinear quark operators $\left(Z_{S}, Z_{P}\right.$, $Z_{V}, Z_{A}$ and $Z_{T}$ ) by using the RI/MOM and RI/SMOM schemes. Our lattice setup is overlap valence quark on $2+1$-flavor domain wall fermion configurations generated by the RBC-UKQCD Collaborations at the physical pion mass $\left[m_{\pi}^{\mathrm{sea}}=139.2(4) \mathrm{MeV}\right]$ [5]. The lattices are of size $48^{3} \times 96$, on which the $\chi$ QCD Collaboration is studying many physical quantities such as vector meson decay constants. We compute $Z_{A}$ from the PCAC relation and obtain the ratios of other RCs to $Z_{A}$ from the appropriate vertex functions. The results are converted to the $\overline{\mathrm{MS}}$ scheme and the scale is set to $2 \mathrm{GeV}$ when there is a scale dependence. These matching factors are necessary to connect the lattice results to the continuum world. For the convenience of later usage we collect our final results in Table IX, where the uncertainties include both the statistical and systematic errors.

The relations $Z_{V}=Z_{A}$ and $Z_{S}=Z_{P}$ for lattice chiral fermions are verified. These relations are better satisfied in the RI/SMOM scheme than in the RI/MOM scheme as shown in Fig. 4 and Fig. 14. This is expected since the

TABLE IX. Matching factors to the $\overline{\mathrm{MS}}$ scheme for the quark field and bilinear quark operators.

\begin{tabular}{lcccc}
\hline \hline$Z_{A}$ & $Z_{q}(2 \mathrm{GeV})$ & $Z_{T}(2 \mathrm{GeV})$ & $Z_{S}(2 \mathrm{GeV})$ & $Z_{P}(2 \mathrm{GeV})$ \\
\hline $1.1025(16)$ & $1.216(23)$ & $1.163(34)$ & $1.118(29)$ & $1.123(56)$ \\
\hline \hline
\end{tabular}


RI/SMOM scheme suppresses more nonperturbative effects by using symmetric momentum configurations.

For $Z_{q}$ and $Z_{T}$, the systematic uncertainties from their conversion ratios to the $\overline{\mathrm{MS}}$ scheme are larger if the $\mathrm{RI} / \mathrm{SMOM}$ scheme is used instead of the RI/MOM scheme. In using both schemes for these two RCs, a renormalization window can be found after the perturbative running to $2 \mathrm{GeV}$, in which a straightforward linear extrapolation in the initial scale $a^{2} p^{2}$ can be done.

For $Z_{S}$ and $Z_{P}$, the conversion ratio from the RI/SMOM scheme to the $\overline{\mathrm{MS}}$ scheme converges much faster than that from the RI/MOM scheme to $\overline{\mathrm{MS}}$. However in using the RI/ SMOM scheme we do not find a broad window in the initial momentum scale for $Z_{S}$ and $Z_{P}$ after the perturbative running, in which both the nonperturbative effects and lattice discretization effects are small. That is to say, unlike for $Z_{q}$ and $Z_{T}$ we do not see a clear linear dependence on $a^{2} p^{2}$ in $Z_{S / P}^{\overline{\mathrm{MS}}}\left(2 \mathrm{GeV} ; a^{2} p^{2}\right)$ after running it from $a^{2} p^{2}$ to $2 \mathrm{GeV}$. We tried several Ansätze equations (44)-(48) to model the behavior of $Z_{S / P}^{\overline{\mathrm{MS}}}\left(2 \mathrm{GeV} ; a^{2} p^{2}\right)$. This leads to the large uncertainties in our calculated $Z_{S}$ and $Z_{P}$ by using the RI/SMOM scheme. This reduced upper edge of the renormalization window may be from the usage of link smearing as discussed in Ref. [27]. We are interested in checking this on thin link configurations in the future.

In Refs. [13,28], two loop calculations of certain Green functions with bilinear quark operator insertions were performed for a general momentum configuration parametrized by $\omega=q^{2} / p^{2}$. For example, $\omega=0$ and 1 correspond to the exceptional and symmetric momentum configuration respectively. The convergence in the perturbative series for the conversion to the $\overline{\mathrm{MS}}$ scheme for different operators behavior differently as a function of $\omega$. Thus, one may want to use a different $\omega$ for a different operator to mostly shrink the truncation error in the conversion ratio.

\section{ACKNOWLEDGMENTS}

We thank RBC-UKQCD Collaborations for sharing the domain wall fermion configurations. This work is partially supported by the National Science Foundation of China (NSFC) under Grants No. 11575197, No. 11575196, No. 11335001, No. 11405178, No. U1632104 and by the U.S. DOE Grant No. DE-SC0013065. Y.C. and Z. L. acknowledge the support of NSFC and DFG (CRC110). This research used resources of the Oak Ridge Leadership Computing Facility at the Oak Ridge National Laboratory, which is supported by the Office of Science of the U.S. Department of Energy under Contract No. DE-AC0500OR22725. This work used Stampede time under the Extreme Science and Engineering Discovery Environment (XSEDE) [29], which is supported by National Science Foundation Grant No. ACI-1053575. We also thank National Energy Research Scientific Computing Center (NERSC) for providing HPC resources that have contributed to the research results reported within this paper. We acknowledge the facilities of the USQCD Collaboration used for this research in part, which are funded by the Office of Science of the U.S. Department of Energy.
[1] M. Gong, A. Alexandru, Y. Chen, T. Doi, S. J. Dong, T. Draper, W. Freeman, M. Glatzmaier, A. Li, K. F. Liu, and Z. Liu, Phys. Rev. D 88, 014503 (2013).

[2] Y.-B. Yang, Y. Chen, A. Alexandru, S.-J. Dong, T. Draper, M. Gong, F. X. Lee, A. Li, K.-F. Liu, Z. Liu, and M. Lujan, Phys. Rev. D 92, 034517 (2015).

[3] Y. Aoki et al. (RBC and UKQCD Collaborations), Phys. Rev. D 83, 074508 (2011).

[4] C. Allton et al. (RBC-UKQCD Collaboration), Phys. Rev. D 78, 114509 (2008).

[5] T. Blum et al. (RBC and UKQCD Collaborations), Phys. Rev. D 93, 074505 (2016).

[6] Y. Chen, A. Alexandru, T. Draper, K. F. Liu, Z. Liu, and Y. B. Yang, arXiv:1507.02541.

[7] R. S. Sufian, Y. B. Yang, A. Alexandru, T. Draper, J. Liang, and K. F. Liu, Phys. Rev. Lett. 118, 042001 (2017).

[8] R. S. Sufian, Y. B. Yang, J. Liang, T. Draper, and K. F. Liu, Phys. Rev. D 96, 114504 (2017).

[9] $\chi \mathrm{QCD}$ Collaboration (to be published).
[10] G. Martinelli, C. Pittori, C. T. Sachrajda, M. Testa, and A. Vladikas, Nucl. Phys. B445, 81 (1995).

[11] Y. Aoki, P. A. Boyle, N. H. Christ, C. Dawson, M. A. Donnellan, T. Izubuchi, A. Jüttner, S. Li, R. D. Mawhinney, J. Noaki, C. T. Sachrajda, A. Soni, R. J. Tweedie, and A. Yamaguchi, Phys. Rev. D 78, 054510 (2008).

[12] C. Sturm, Y. Aoki, N. H. Christ, T. Izubuchi, C. T. C. Sachrajda, and A. Soni, Phys. Rev. D 80, 014501 (2009).

[13] M. Gorbahn and S. Jäger, Phys. Rev. D 82, 114001 (2010).

[14] L. G. Almeida and C. Sturm, Phys. Rev. D 82, 054017 (2010).

[15] H. Neuberger, Phys. Lett. B 417, 141 (1998).

[16] T.-W. Chiu and S. V. Zenkin, Phys. Rev. D 59, 074501 (1999).

[17] Z. Liu, Y. Chen, S.-J. Dong, M. Glatzmaier, M. Gong, A. Li, K.-F. Liu, Y.-B. Yang, and J.-B. Zhang (chiQCD Collaboration), Phys. Rev. D 90, 034505 (2014).

[18] C. Wang, Y. Bi, H. Cai, Y. Chen, M. Gong, and Z. Liu, Chin. Phys. C 41, 053102 (2017).

[19] K. G. Chetyrkin and A. Retey, Nucl. Phys. B583, 3 (2000).

[20] A. I. Alekseev, Few-Body Syst. 32, 193 (2003). 
[21] T. van Ritbergen, J. A. M. Vermaseren, and S. A. Larin, Phys. Lett. B 400, 379 (1997).

[22] C. Patrignani et al. (Particle Data Group), Chin. Phys. C 40, 100001 (2016).

[23] K. G. Chetyrkin, Phys. Lett. B 404, 161 (1997).

[24] J. A. Gracey, Nucl. Phys. B662, 247 (2003).

[25] J. A. Gracey, Eur. Phys. J. C 71, 1567 (2011).

[26] T. Blum, N. Christ, C. Cristian, C. Dawson, G. Fleming, G. Liu, R. Mawhinney, A. Soni, P. Vranas, M. Wingate,
L. Wu, and Y. Zhestkov, Phys. Rev. D 66, 014504 (2002).

[27] R. Arthur, P. A. Boyle, S. Hashimoto, and R. Hudspith, Phys. Rev. D 88, 114506 (2013).

[28] J. M. Bell and J. A. Gracey, Phys. Rev. D 93, 065031 (2016).

[29] J. Towns, T. Cockerill, M. Dahan, I. Foster, K. Gaither, A. Grimshaw, V. Hazlewood, S. Lathrop, D. Lifka, G. D. Peterson, R. Roskies, J. Ray Scott, and N. Wilkins-Diehr, Comput. Sci. Eng. 16, 62 (2014). 